\title{
Informational Paradigm, management of uncertainty and theoretical formalisms in the clustering framework: A review
}

\author{
Pierpaolo D'Urso \\ Department of Social Sciences and Economics, Sapienza Università di Roma, P.za Aldo Moro, 5, 00185, Rome, Italy
}

\section{A R T I C L E I N F O}

\section{Article history:}

Received 16 November 2016

Revised 23 January 2017

Accepted 1 March 2017

Available online 3 March 2017

\section{Keywords:}

Statistical reasoning

Informational Paradigm

Empirical and theoretical information

Uncertainty associated with empirical and

theoretical information

Uncertainty managing

Uncertainty formalisms

Fuzzy clustering

Possibilistic clustering

Shadowed clustering

Rough sets-based clustering

Intuitionistic fuzzy clustering

Evidential clustering

Credibilistic clustering

Type-2 fuzzy clustering

Neutrosophic clustering

Hesitant fuzzy clustering

Interval-based fuzzy clustering

Picture fuzzy clustering

\begin{abstract}
A B S T R A C T
Fifty years have gone by since the publication of the first paper on clustering based on fuzzy sets theory. In 1965, L.A. Zadeh had published "Fuzzy Sets" [335]. After only one year, the first effects of this seminal paper began to emerge, with the pioneering paper on clustering by Bellman, Kalaba, Zadeh [33], in which they proposed a prototypal of clustering algorithm based on the fuzzy sets theory. Starting from this paper, several uncertain clustering methods based on different theoretical approaches for modeling the uncertainty have been proposed. The present paper presents a systematic literature review of these clustering approaches. In particular, with respect to the Statistical Reasoning System, we first illustrate the connection between Information and Uncertainty from the perspective of the so-called Informational Paradigm, according to which Information is constituted by "Informational ingredients", specifically the "Empirical Information," represented by statistical data, and "Theoretical information" consisting of background knowledge and basic modeling assumptions. We then describe different kinds of uncertainty affecting the Information. Focusing on the uncertainty associated with a particular statistical methodology, i.e. Cluster Analysis, and adopting as theoretical platform the Informational Paradigm, we present a systematic literature review of different uncertainty-based clustering approaches -i.e. Fuzzy clustering, Possibilistic clustering, Shadowed clustering, Rough sets-based clustering, Intuitionistic fuzzy clustering, Evidential clustering, Credibilistic clustering, Type-2 fuzzy clustering, Neutrosophic clustering, Hesitant fuzzy clustering, Interval-based fuzzy clustering, and Picture fuzzy clustering. We thus show how all these clustering approaches are able of managing in different ways the uncertainty associated with the two components of the Informational Paradigm, i.e. the Empirical and Theoretical Information.
\end{abstract}

(c) 2017 Elsevier Inc. All rights reserved.

\section{On the 50th anniversary of fuzzy clustering}

In the knowledge discovery process, although the Statistical Reasoning System

may be efficient a halo of uncertainty always will permeate

the information and therefore the knowledge;

the only certainty is that there are no certainties.

E-mail address: pierpaolo.durso@uniroma1.it 


\section{Introduction}

Statistical reasoning can be viewed as a specific instance of approximate reasoning, where uncertainty affects the various ingredients of the reasoning process, which is therefore characterized by "approximation."

In particular, Statistical Reasoning Systems embody two types of "informational" ingredients, the Empirical Information represented by the dataset, and the initial Theoretical Information, which includes basic modeling assumptions, previous knowledge, and other pieces of Theoretical Information concerning the processing assumptions and the cognitive conclusions of the knowledge acquisition process (the "informational gain" obtained by means of appropriate strategies of analysis applied in the above context).

All of the above informational ingredients are affected by some source of uncertainty. For example, the data may be imprecisely measured or vaguely defined (e.g. use of linguistic expressions); furthermore, they may only partially represent the universe of possible data describing the investigated phenomenon (for instance when they are sampled from a larger population). Moreover, the basic modeling assumptions may also be uncertain, and the same is true of the assumptions used for processing the data (in fact any particular specification of these assumptions involves uncertainty as to their validity in the given research framework). Finally, the results of the statistical analysis reflect the uncertainties associated with the various pieces of information used for drawing the conclusions. In this respect, we have to cope with an uncertainty propagation process matching a parallel information propagation process, within the same Statistical Reasoning System.

In the above framework, randomness, imprecision, vagueness, partial ignorance are different types of uncertainty requiring a specific treatment. Standard probability theory may not be sufficient for dealing with all of them. We argue that fuzzy sets theory, as well as other uncertainty theories -such as, e.g., Type-2 fuzzy sets theory, Intuitionistic fuzzy sets theory, Rough sets theory, Shadowed sets theory, Credal sets theory and Evidential theory, Possibility and Credibilistic theories, Neutrosophic sets theory, Hesitant sets theory or inferential logic based on conditional probability (seen as a function of the conditioning event)- can suitably integrate the traditional probability theory in order to deal with the complexity of statistical reasoning.

In this connection, the paper will focus on the specific area of Cluster Analysis to illustrate the different theoretical approaches used in the literature to manage the uncertainty in the clustering process.

Fifty years have gone by since the publication of the first paper on clustering, based on fuzzy sets theory. In 1965, L.A. Zadeh had published "Fuzzy Sets" [332]. After only one year, the first effects of this seminal paper began to emerge, with the pioneering paper on clustering by Bellman, Kalaba, Zadeh [33], in which they proposed a prototypal of clustering algorithm based on the fuzzy sets theory. Starting from this paper, several uncertain clustering methods based on different theoretical approaches for modeling the uncertainty have been proposed.

The present paper presents a systematic literature review of these clustering approaches.

In particular, the main aim of the paper is to show in an organic manner the impressive impact of the seminal papers on fuzzy clustering on different scientific communities- mathematicians, statisticians, computer scientists, and so on- in the last 50 years. In fact, as we can see below, a massive and diversified scientific production has characterized those fruitful years. To do this, we define a general theoretical platform, i.e. the so-called Informational Paradigm, to manage different kinds of information and uncertainty, organically interconnected, which characterize the Statistical reasoning methods and in particular the clustering processes. Thus, we analyze systematically and in detail the Informational Paradigm, the possible uncertainty affecting different kinds of information and the connected theoretical formalisms for managing in a different manner the uncertainty, focusing on the fuzzy set theory and on its more fruitful theoretical extensions and generalizations in a methodological point of view. Successively, we adopt the Informational Paradigm as theoretical platform for the clustering methodology, showing different approaches for managing the uncertainty in the classification process. In this way, we assume that the different uncertainty-based clustering approaches are defined on the basis of the Information Paradigm. Thus, in different sections of the paper, we review systematically and in detail the more relevant uncertainty-based clustering approaches proposed in the literature for classifying objects and explain the respective theories used for managing the uncertainty. For each clustering approach, we illustrate the chronology of the various theoretical and methodological contributes, showing, with respect to the Informational Paradigm, the information ingredients and the uncertainty measures connected to different clustering approaches. Furthermore, we compare in a chronological point of view the different uncertainty-based clustering approaches and the connected uncertainty theories, showing the different timing of the impacts of the various uncertainty theories from the respective clustering approaches and then the different metabolic process of the theoretical results used in the respective clustering methodologies.

The paper is organized as follows. Starting from the definition of Informational Paradigm (Section 2), we illustrate various non probabilistic formalisms for managing uncertainty in data analysis (Section 3), including fuzzy sets theory and its developments, and theories that manage imprecision and uncertainty in a different way. Focusing on the fuzzy sets theory and on some of its recent developments -i.e. the Type-2 fuzzy sets theory, Intuitionistic fuzzy sets theory, Rough sets theory, Shadowed sets theory, Credal sets theory and Evidential theory, Possibility and Credibilistic theories, Neutrosophic sets theory, Hesitant sets theory and Picture Fuzzy Sets- in Section 4, we present a review of clustering methods based on the various formalisms present in the literature. As we shall see, these formalisms manage in different ways the uncertainty associated with the two components of the Informational Paradigm, i.e. the Empirical and Theoretical Information. A summary and some conclusions are presented, respectively in Sections 5 and 6. 


\section{Informational Paradigm and uncertainty}

Following the general approach to Statistical Reasoning called "Informational Paradigm" [61,62] the Information (I) is made up of "Informational Ingredients", which include the "Empirical Information" $\left(\mathrm{I}_{\mathrm{E}}\right)$, i.e. the statistical data, and by "Theoretical information" $\left(\mathrm{I}_{\mathrm{T}}\right)$ i.e. the background knowledge and basic modeling assumptions. The cognitive conclusions of the process constitute the "Informational Gain" $\left(\mathrm{I}_{G}\right)$ of the Statistical Reasoning System.

All of these informational ingredients can be affected by various kinds of Uncertainty (U). A reasonable list is the following:

- The uncertainty of the relation between the observed data and the "universe" of possible data (in the traditional inferential statistical approach this is managed by means of a probabilistic sampling model).

- The uncertainty associated with the different components of a statistical method: algorithm, procedure, model and so on.

- The margin of error in the measurements of the empirical phenomena.

- The vagueness of the linguistic terms describing the phenomena.

- The partial or total ignorance of a phenomenon in specific observational instances (e.g. missing data), or of the underlying theoretical assumptions.

- The uncertainty deriving from the "granularity" of the terms used in the description of the physical world (this is related to the general notion of "linguistic variable" whose "granules" are "clumps of values drawn together by indistinguishability, similarity, proximity or functionality"). Note that this notion is more general than that of qualitative variable used in Statistics. Specifically, it refers to general cognitive processes, including the definition and use of models, theoretical assumptions, etc., and therefore, also to what we called theoretical information in statistical reasoning.

If we view Statistical Reasoning from the perspective of the Informational Paradigm, we can distinguish two parallel processes: on one side the manipulation of the Informational ingredients (from the Initial Information (I), through the Processing Assumptions and the final Informational Gain); on the other side the propagation of Uncertainty (from the initial Uncertainties $(\mathrm{U})$, through the Uncertainties associated with the various Informational ingredients introduced into the Statistical Reasoning process, i.e. $U_{E}$ (the Uncertainty associated with $\mathrm{I}_{\mathrm{E}}$ ) and $\mathrm{U}_{\mathrm{T}}$ (the Uncertainty associated with $\mathrm{I}_{\mathrm{T}}$ ), up to the final assessment of Uncertainty related to the Additional Information $\left(\mathrm{I}_{\mathrm{A}}\right)$ obtained by the given Statistical Reasoning System, i.e. Final Uncertainty $\left(\mathrm{U}_{\mathrm{F}}\right)$, associated with the Final Information $\left(\mathrm{I}_{\mathrm{F}}\right)$ obtained manipulating and combining suitably the two informational entities of the Informational Paradigm). Note that, by manipulating/combining the informational ingredients, we intrinsically manage the uncertainty obtaining suitable uncertainty measures capable of improving and increasing information. Therefore, Information and Uncertainty appear to be strictly linked with one another, i.e. information and uncertainty are organically interconnected, so that we can speak of "Information-based Uncertainty" as well as of "Uncertainty based Information" [147]. For more details, see Coppi [61,62] and Coppi et al. [64].

In Fig. 1, we schematically present these two processes.

The different kinds of uncertainty associated with the two types of information can be modeled following different theoretical formalisms. In the following section, we will consider the formalization of the uncertainty in a non-probabilistic manner, by considering the fuzzy sets theory introduced by Lofti A. Zadeh in 1965 [332]. In addition, in Section 3, we will illustrate different developments of Zadeh's theory.

\section{Management of uncertainty in the Informational Paradigm: fuzzy sets theory and other theoretical formalisms}

In the scientific literature of the twentieth-century, a few theoretical frameworks for developing Statistical Reasoning have been suggested -from the classical Inferential Paradigm (e.g, [67]) to the Descriptive-Exploratory Paradigm of the French school usually referred to as "Analyze des Données" (e.g., [34]), to the more recent Statistical Learning Paradigm (e.g., Vapnik, [295]). However, all of the above frameworks do not allow for a complete treatment of the various sources of Uncertainty affecting the Statistical Reasoning process. In fact, the main source of Uncertainty investigated in the above mentioned theoretical frameworks is "randomness," quite often limited to the "data generation process" managed by means of appropriate probabilistic models [62]. In this area, Zadeh's fundamental contribution (1965), by introducing the notion of Fuzzy Sets, opened the way to a new development of logical, mathematical and statistical thinking. In close connection with Probability Theory, Fuzzy Sets Theory may provide the necessary tools for a generalized treatment of Uncertainty in Statistics [62] and powerful tools for expressing and managing some of the abovementioned uncertainties and the associated informational entities beyond the traditional domains of probability theory in its standard form [64].

\subsection{Fuzzy sets theory and other theoretical formalisms}

In the classical Set Theory introduced by G. Cantor in 1874, the membership of elements in a set is assessed in binary terms according to a Principle of Bivalence: an element either belongs or does not belong to the set. By contrast, in the fuzzy set theory proposed by L.A. Zadeh [332], the membership of elements in a set is assessed in gradual terms, and can be described with the aid of a Membership function valued in the real unit interval $[0,1]$. Thus, fuzzy sets represent a 
Initial Information

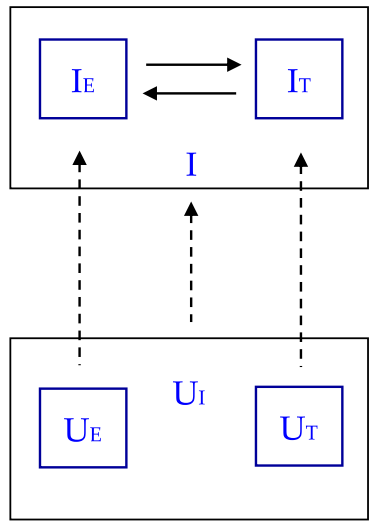

Initial Global Uncertainty
Final Information

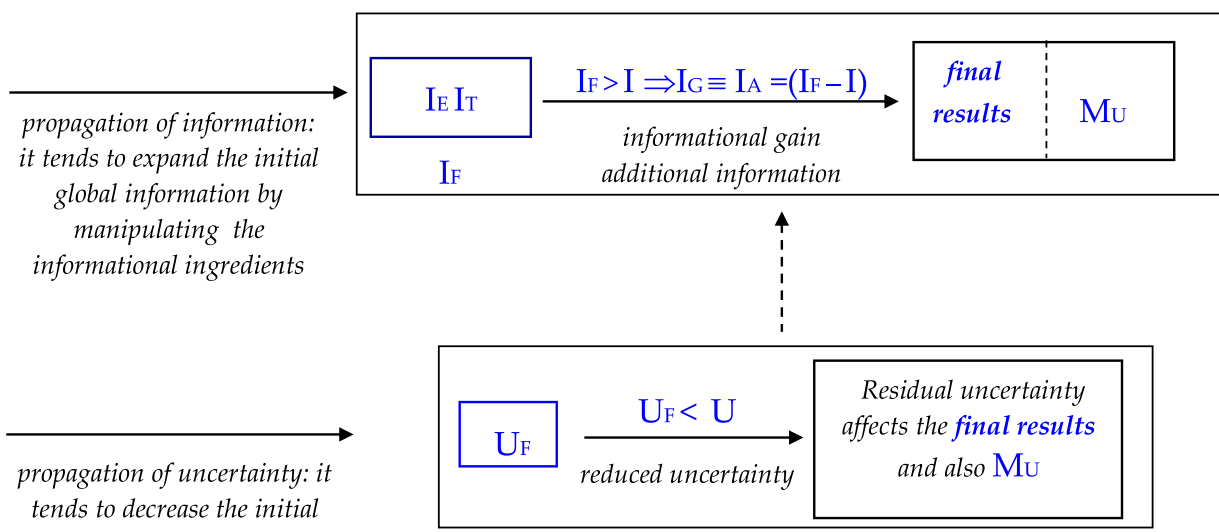

Final Uncertainty
$\mathrm{IE}=$ Empirical Information

$\mathrm{I}_{\mathrm{T}}=$ Theoretical Information

$\mathrm{I}=$ Initial Information or Information

$\mathrm{I}_{\mathrm{F}}=\mathrm{IE}_{\mathrm{E}} \mathrm{I}_{\mathrm{T}}=$ Final Information

$\mathrm{IG}_{\mathrm{G}}=$ Informational Gain

$\mathrm{I}_{\mathrm{A}}=$ Additional Information

\footnotetext{
$\mathrm{U}_{\mathrm{E}}=$ Uncertainty associated with $\mathrm{IE}_{\mathrm{E}}$

$\mathrm{U}_{\mathrm{T}}=$ Uncertainty associated with $\mathrm{I}_{\mathrm{T}}$

$\mathrm{U}=$ Uncertainty associated with $\mathrm{I}$

$U_{\mathrm{F}}=$ Uncertainty associated with $\mathrm{IF}_{\mathrm{F}}$

$\mathrm{Mu}=$ Measures of Uncertainty
}

Fig. 1. Scheme of the Informational Paradigm and uncertainty.

generalization of classical sets, since the Indicator function of classical sets are special cases of the membership functions of fuzzy sets, in which membership can only take the values 0 or 1 . In fuzzy set theory, classical bivalent sets are usually called "Crisp sets."

In the literature on the management of uncertainty from a non-exclusively probabilistic perspective, fuzzy sets theory represents a crucial innovation. Indeed, starting from this theory a great number of theoretical formalisms for managing in different ways the various kinds of uncertainty affecting the two informational entities of the Informational Paradigm (i.e. the empirical and theoretical information) have been suggested, and many new mathematical constructions and theories treating imprecision, inexactness, ambiguity, vagueness, and uncertainty have been developed. Some of these constructions and theories are extensions of fuzzy set theory, while others try to mathematically model imprecision and uncertainty in different ways (Burgin Chnihin [361] $[99,144]$ ). Note that while most of the above can be generally categorized as truthbased extensions of fuzzy sets, bipolar fuzzy set theory presents a philosophically and logically different, equilibrium-based generalization of fuzzy sets [339-341].

The most relevant theoretical formalisms are listed in Table 1 and in the timeline illustrated in Fig. 2 (in the list, we also include sets and fuzzy sets theories).

Some of the formalisms shown in Table 1 have been utilized for managing uncertainty in specific domains of Statistics and in particular in Cluster Analysis. In this connection, in the following section, we will illustrate a detailed review of the clustering approaches used to appropriately deal with different kinds of uncertainty in clustering processes.

\section{Informational Paradigm and different uncertainty formalisms in the clustering approach}

The Informational Paradigm can be applied to all forms of Statistical Reasoning; in particular, we focus our attention on cluster analysis.

For example, in a non-hierarchical clustering framework, the general optimization problem associated to the clustering process can be formalized as follows:

$$
\left\{\begin{array}{l}
\text { Optimization (with respect to } u \text { and } h \text { ) of the objective function }: g(x ; u, h ; c, m, d) \\
\text { subject to possible constraints }
\end{array}\right.
$$

where:

$g=$ type of objective function, $h=$ kind of cluster prototype, $u=$ uncertainty measure, $x=$ input data, $c=$ number of clusters, $m=$ parameter for tuning suitably the uncertainty, $d=$ distance measure. 


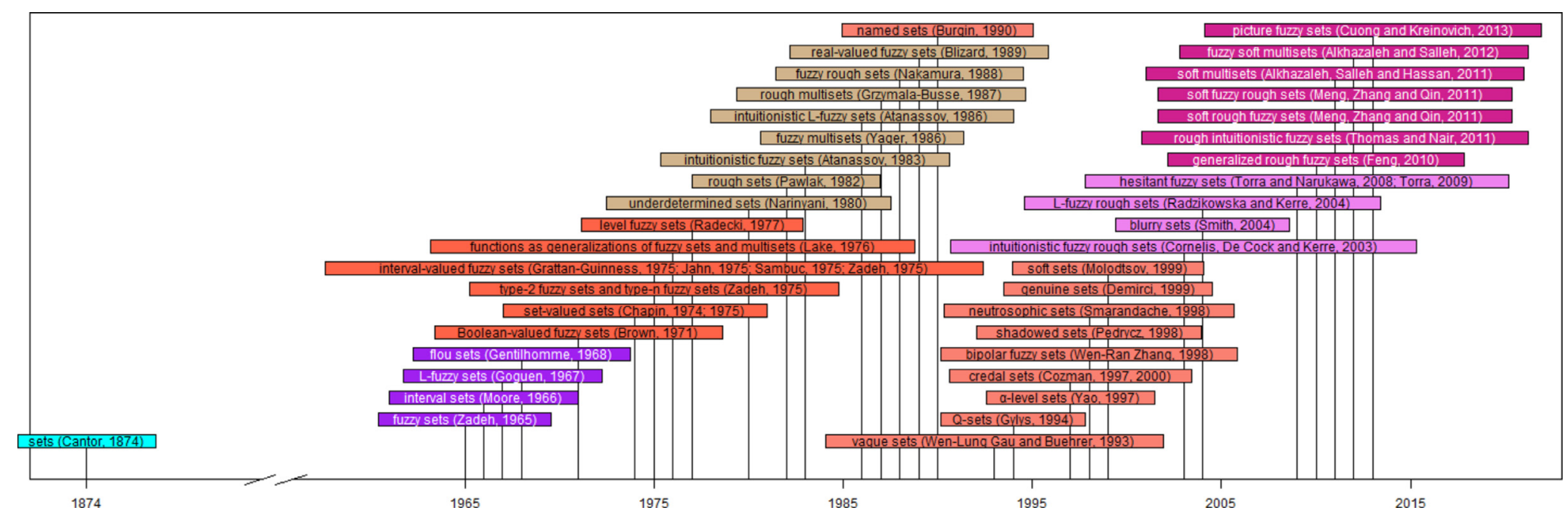

Fig. 2. Timeline of theoretical formalisms (note that theories labeled with the same color refer to the same decade) 
Table 1

Theoretical formalisms for managing the uncertainty $(*)$.

\begin{tabular}{|c|c|}
\hline 1. Sets [45] & 25. bipolar fuzzy sets [340] \\
\hline 2. fuzzy sets [332] & 26. shadowed sets [225] \\
\hline 3. interval sets [211] & 27. neutrosophic sets [274] \\
\hline 4. L-fuzzy sets [112] & 28. genuine sets [89] \\
\hline 5. flou sets [110] & 29. soft sets [210] \\
\hline 6. Boolean-valued fuzzy sets [38] & 30. intuitionistic fuzzy rough sets $[65,66]$ \\
\hline 7. set-valued sets $[50,51]$ & 31. blurry sets [276] \\
\hline 8. type-2 fuzzy sets and type-n fuzzy sets [353] & 32. L-fuzzy rough sets [249] \\
\hline 9. interval-valued fuzzy sets $[115,132,263,353]$ & 33. hesitant fuzzy sets [290] \\
\hline 10. functions as generalizations of fuzzy sets and multisets [159] & 34. generalized rough fuzzy sets [106] \\
\hline 11. level fuzzy sets [248] & 35. rough intuitionistic fuzzy sets [283] \\
\hline 12. underdetermined sets [213] & 36. soft rough fuzzy sets [196] \\
\hline 13. rough sets [221] & 37. soft fuzzy rough sets [196] \\
\hline 14. intuitionistic fuzzy sets [25] & 38. soft multisets [7] \\
\hline 15. fuzzy multisets [354] & 39. fuzzy soft multisets [8] \\
\hline 16. intuitionistic L-fuzzy sets [25] & 40. picture fuzzy sets [73] \\
\hline \multicolumn{2}{|l|}{ 17. rough multisets [117] } \\
\hline \multicolumn{2}{|l|}{ 18. fuzzy rough sets $[212]$} \\
\hline \multicolumn{2}{|l|}{ 19. real-valued fuzzy sets [37] } \\
\hline \multicolumn{2}{|l|}{ 20. named sets [39] } \\
\hline \multicolumn{2}{|l|}{ 21. vague sets [109] } \\
\hline \multicolumn{2}{|l|}{ 22. Q-sets [122] } \\
\hline \multicolumn{2}{|l|}{ 23. $\alpha$-level sets $[325,326]$} \\
\hline 24. credal sets $[68,69]$ & \\
\hline
\end{tabular}

(*) Note that, for completeness in the list we consider the sets theory; however, it represents the logicmathematical construction basis for modeling the uncertainty in a probabilistic perspective by means of the probabilistic theory.

Thus, from an informational perspective, the informational ingredients $I_{E}$ and $I_{T}$ of the Informational $I$ and the Final Information are:

$$
\begin{aligned}
& I \equiv\left\{I_{E}=(x), I_{T}=(\text { optimization criterion, } g(.), c, m, d, \text { type of prototype })\right\} \\
& I_{F} \equiv\{\text { results }: u, h\} .
\end{aligned}
$$

The initial uncertainty $(U)$ associated to I concerns, respectively, the nature of the input data $x\left(U_{E}\right)$ and the theoretical ingredients of the clustering method (i.e. the optimization criterion, $g(), c, m,$.$d and the type of prototype) \left(U_{T}\right)$; the final uncertainty is related to the computed results and their interpretation $\left(\mathrm{U}_{\mathrm{F}}\right)$.

Note that, by manipulating and combining the informational ingredients, we intrinsically manage the uncertainty, obtaining suitable uncertainty measures capable of improving and increasing the informational power associated with the clustering process. The uncertainty measurements are represented directly by $u$ and/or their elaborations.

Note that, since according to the Informational Paradigm the final information is also affected by uncertainty, the computed point uncertainty measurement $u$ (a piece of the final information) is itself affected by uncertainty; therefore, in the knowledge discovery process, the Statistical Reasoning Systems is not able to completely eliminate uncertainty. No matter how efficient the system is, a halo of uncertainty always permeates the information.

From this perspective, in Cluster Analysis there are different theoretical approaches for properly managing the different kinds of uncertainty affecting the Empirical and Theoretical Information of the Information Paradigm, i.e., respectively, the data and the various theoretical ingredients of the clustering methods.

In terms of managing uncertainty through a statistical and probabilistic approach, there are several methods based on the use of finite mixture models for clustering of multivariate data observed from a random sample. Such models can be fitted by maximum likelihood via the Expected-Maximization (EM) algorithm (see, among others, [193]).

The most used non-probabilistic approach for managing different kinds of uncertainty in the clustering framework is the fuzzy sets theory [332]. The literature on fuzzy clustering is boundless. As we can see in Section 4.1, in the early methods (see, e.g., Bezdek, [355]), uncertainty is modeled exclusively with respect to the theoretical component of the Informational Paradigm, by means of the concept of membership degree, represented by an uncertainty measurement in the assignment process of objects to different clusters.

In the literature, there are also several methods in which uncertainty is modeled by considering simultaneously the random (stochastic) and fuzzy approach, by means of the theory of Fuzzy Random Variables (FRVs). From a theoretical viewpoint, this implies constructing appropriate probability measurements of fuzzy sets. Various proposals have been made, in the last decades, within this perspective. One of the most significant proposals is the notion of FRV as introduced by Puri and Ralescu [245] and Klement et al. [146]. Another definition for a fuzzy-valued random variable which is mathematically equivalent, albeit conceptually different and can only be stated for the univariate case, is the one proposed by Kwakernaak [154,157] and Kruse and Meyer [153]. For an example of clustering based on FRVs see, Colubi et al. [59].

In the following sections, we will focus our attention on the clustering approaches based on the fuzzy sets theory and on different extensions and derivatives of this prolific theory. 
In particular, we present a literature review of the following uncertainty-based clustering approaches (ordered with respect to the first published paper of each approach):

- Fuzzy clustering,

- Possibilistic clustering,

- Shadowed clustering,

- Rough sets-based clustering,

- Intuitionistic fuzzy clustering,

- Evidential clustering or credal clustering or Belief clustering,

- Credibilistic clustering,

- Type-2 fuzzy clustering,

- Neutrosophic clustering,

- Hesitant fuzzy clustering,

- Interval-based fuzzy clustering,

- Picture fuzzy clustering.

\subsection{Fuzzy clustering}

Fuzzy Sets (FSs), as already mentioned, is a powerful theory introduced by Zadeh [332] more than 50 years ago for suitably managing uncertainty and imprecision in knowledge discovery processes. Mathematically, a Fuzzy Set (FS) (or Type1 Fuzzy Set, T1FS) can be defined as follows.

Let $\mathrm{X}$ be an initial universe. A Fuzzy Set (FS) (or T1FS) $\mathrm{A} \subseteq \mathrm{X}$ is defined as follows:

$$
\mathrm{A}=\left\{\left(\mathrm{X}, \mu_{\mathrm{A}}(\mathrm{x}): \mathrm{x} \in \mathrm{X}\right\}\right.
$$

where $\mu_{\mathrm{A}}(\mathrm{x})$ is called "membership function" of $\mathrm{x}$ to $\mathrm{A}$ with $\mu_{\mathrm{A}}(\mathrm{x}) \in[0,1]$.

The definition of a FS is a simple extension of the definition of a classical set in which the membership function $\mu_{\mathrm{A}}(\mathrm{x})$ is restricted to either 0 or 1 , i.e. $\mu_{A}(\mathrm{x}) \in\{0,1\}$.

Fuzzy Sets theory immediately showed its theoretical and applicative potential in the cluster analysis in which it was widely used.

Traditional clustering methods mandate that an object must belong to precisely one cluster. Such a requirement is found to be too restrictive in many applications. In practice, an object may display characteristics of different clusters. In such cases, an object should belong to more than one cluster and as a result, cluster boundaries necessarily overlap. The fuzzy set representation of clusters makes it possible for an object to belong to multiple clusters with a membership degree between 0 and 1 [169].

In Statistical Reasoning, clustering constitutes the first statistical technique which lends itself to a treatment based on the fuzzy sets theory [332]. The rationale for it lies in the recognition of the vague nature of the cluster assignment task. In the literature, several fuzzy clustering methods have been proposed and applied in many different fields [78].

As already mentioned, the pioneering fuzzy clustering algorithm was proposed in 1966 by Bellman, Kalaba, Zadeh [33]. Successively, other authors -i.e. Wee [306], Flake and Turner (1968), Gitman and Levine [111] and Ruspini [260-262]- contributed to the original clustering algorithm based on fuzzy sets theory. As remarked by Bezdek in his first paper on fuzzy clustering, the previous papers "trace the evolution of fuzzy sets as a theoretical basis for cluster analysis" [35]. With regard to Ruspini's approach, however, "the original algorithm [...] is said to be rather difficult to implement. Its computational efficiency should be weak and its generalization to more than two clusters should be of little success. But it was the pioneer for a successful development of this approach" [28]. Ruspini's method opened the door for further research, especially thanks to the idea of fuzzy c-partitions in cluster analysis [357].

The Fuzzy c-Means (FcM) clustering method independently introduced by Dunn [356] and Bezdek [35] and then analytically formalized and investigated from a more general perspective by Bezdek [355], is the first method which is computationally efficient and powerful. For this reason, the Bezdek's method represents the best-known and used clustering technique in the body of literature. Several fuzzy approach-based clustering methods have been developed by extending suitably the original Bezdek's method proposed in the 1981 [78].

Let $\mathbf{X}=\left\{x_{i s}: i=1, \ldots, n ; s=1, \ldots, p\right\}=\left\{\mathbf{x}_{i}=\left(x_{i 1}, \ldots, x_{i s}, \ldots, x_{i p}\right)^{\prime}: i=1, \ldots, n\right\}$ be the data matrix, where $x_{i s}$ represents the sth variable observed on the $i$ th object and $\mathbf{x}_{i}$ represents the vector of the $i$ th observation.

The FcM clustering method proposed by Bezdek [355] can be formalized in the following way (for the iterative solutions see Bezdek, [355]):

$$
\begin{aligned}
& \min : \sum_{i=1}^{n} \sum_{k=1}^{c} u_{i k}^{m} d^{2}\left(\mathbf{x}_{i}, \mathbf{h}_{k}\right) \\
& \sum_{k=1}^{c} u_{i k}=1, u_{i k} \geq 0
\end{aligned}
$$

where $u_{i k}$ indicates the membership degree of the $i$ th object to the $k$ th cluster; $d^{2}\left(\mathbf{x}_{i}, \mathbf{h}_{k}\right)=\left\|\mathbf{x}_{i}-\mathbf{h}_{k}\right\|^{2}$ represents the squared Euclidean distance between the $i$ th object and the centroid of the $k$ th cluster, i.e. $\mathbf{h}_{k}=\left(h_{k 1}, \ldots, h_{k s}, \ldots, h_{k p}\right)^{\prime}$ repre- 
sents the $k$ th centroid, where $h_{k s}$ indicates the $s$ th component (sth variable) of the $k$ th centroid vector; $m>1$ is a parameter that controls the fuzziness of the partition.

With respect to the Informational Paradigm $(I)$ and the informational ingredients of the FcM clustering method (1), we have that the Empirical Information $\left(I_{E}\right)$ is represented by $\mathbf{x}_{i}(i=1, \ldots n)$ (the elements of the data matrix) and the Theoretical Information $\left(I_{T}\right)$ is represented by theoretical informational ingredients of the clustering method, i.e., minimization criterion, additive quadratic objective function, $c$ (number of clusters) and $m$ (fuzziness parameter), $d^{2}$ (.) (squared Euclidean distance measure), type of prototype (centroid); the final information is constituted by $u_{i k}(i=1, \ldots n ; k=1, \ldots, c)$ (the membership degrees of the object to the clusters), $\mathbf{h}_{k}(k=1, \ldots, c)$ (the centroids of the clusters). In symbol, we have:

$I \equiv\left\{I_{E}=\left(\mathbf{x}_{i}\right), I_{T}=\right.$ (minimization criterion, additive quadratic objective function, $c, m$, squared Euclidean distance measure, type of prototype): $i=1, \ldots, n\}$

$I_{F} \equiv\left\{\right.$ results: $\left.u_{i k}, \mathbf{h}_{k}: i=1, \ldots n ; k=1, \ldots, c\right\}$.

Thus, uncertainty can affect all the previous components of the Informational Paradigm.

By considering the clustering version proposed by Bezdek [355] the uncertainty is measured directly by $u_{i k}(i=1, \ldots . n$; $k=1, \ldots, c)$, i.e. we consider only the uncertainty associated with the assignment process of the objects to the clusters; no other kinds of uncertainty are taken into account in the original FcM clustering method. In particular, $u_{i k}(i=1, \ldots . n ; k=1, \ldots, c)$ represents a point measure of the uncertainty in the sense that for each membership degree we have a single value representing the measurement of the uncertainty in the assignment process of each object to each cluster. Derivative uncertainty measurements can be obtained from $u_{i k}$ defining suitable within and between variability measures of the clusters of the computed partition.

In the literature, there are several extensions of (1) in which the Empirical Information (represented by the input data) is affect by uncertainty, imprecision, vagueness and so on.

In particular, by considering the case in which the imprecision of the data are formalized in a fuzzy manner (fuzzy data) we point out the following contributes on fuzzy clustering of fuzzy data: Sato and Sato [266], Hathaway et al. [124], Yang and Ko [320], Yang and Liu [321], Takata et al. [282], Auephanwiriyakul and Keller [26], Butkiewicz [41], Hung and Yang [128], D’Urso and Giordani [80], Zarandi and Razaee [334], Coppi et al. [63]. Suggestive applications of fuzzy clustering methods for fuzzy data in e-health and tourism have been suggested, respectively, by D'Urso et al. [74] and D'Urso et al. [76,78]. Recently, robust fuzzy clustering methods for fuzzy data have been suggested by D'Urso and De Giovanni [77]. They, using a "Partitioning Around Medoids" (PAM) approach, firstly proposed a timid robustification of the fuzzy clustering for LR fuzzy data; successively, proposed three robust fuzzy clustering models based, respectively, on noise cluster, exponential metric and trimming rules. For a survey on fuzzy clustering of fuzzy data, see D'Urso [75].

In the literature, there are also various clustering methods for classifying imprecise data modeled as interval-valued data. See, e.g., El-Sonbaty and Ismail (1998), D’Urso and Giordani [80,81], de Carvalho [86], de Carvalho and Tenorio [85], D’Urso et al. [105], D’Urso and Leski [82].

An interesting approach for managing the imprecision affecting the data is the granular computing [29,228,233] As remarked by Zadeh [331] wrote "fuzzy information granulation underlies the remarkable human ability to make rational decisions in an environment of imprecision, partial knowledge, partial certainty and partial truth" . In this respect, in a clustering framework, in terms of granular computing, a cluster can be interpreted as an information granule that presents its objects on a coarser and more granular level [107]. Useful references on clustering of granular data are, e.g., [104,107,168,231,232,241,265].

In addition, in the literature there are many methods based on extensions or alternatives of the objective function represented in (1) (e.g. methods with entropy regularization, relational methods, hybrid methods, robust methods and so on). In all these cases, the new additional parameters considered in the respective objective functions represent other ingredients of the Theoretical Information, themselves affected by uncertainty.

For interested readers, useful references to the extensive literature on fuzzy clustering are found in the chapter on the fuzzy clustering by D'Urso in the Handbook of Cluster Analysis [42], the seminal monograph by Bezdek [355], the books by Jain, Dubes [133], de Oliveira, Pedrycz [87], Miyamoto, Ichihashi, Honda [207] and, e.g., the following journals devoted to this topic: Fuzzy Sets and Systems, IEEE Transactions on Fuzzy Systems, Information Sciences, Pattern Recognition, Applied Soft Computing, Soft Computing, Pattern Recognition Letters.

\subsection{Possibilistic clustering}

In FcM (see (1)), for each object, the sum of the membership degrees in the clusters must be equal to one. Such a constraint may cause meaningless results, especially when noise is present. Following the Possibility Theory [333], to avoid this drawback, it is possible to relax the constraint, leading to the so-called Possibilistic c-Means (PcM) clustering, providing "degrees of compatibility" of an object with each of the clusters [78]. Note that, in general the Possibility Theory is related to the Fuzzy Sets Theory by defining the concept of a possibility distribution as a fuzzy restriction which acts as an elastic constraint on the value that may be assigned to a variable [333]. A revised fuzzy set interpretation of possibility theory was introduced by Klir [148].

In the possibilistic perspective, $u_{i k}$ represents the degree of possibility of object $i$ belonging to cluster $k$ or, in other terms, the degree of compatibility of the profile $\boldsymbol{x}_{i}$ with the characteristics of cluster $k$ embodied by its prototype $\mathbf{h}_{k}$. The 


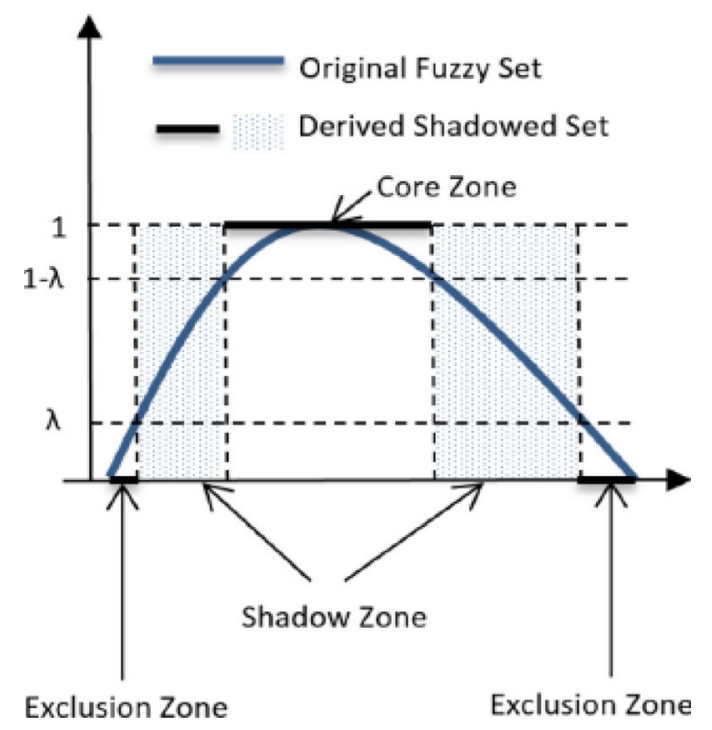

Fig. 3. Three zones in shadowed set divided by the threshold $\lambda$ (source: [337]).

FcM objective function is consequently modified, by introducing an additive "penalization" term which takes care of the balance between the fuzziness of the clustering structure and the "compactness" of the clusters [78].

The first PcM clustering method was proposed by Krishnapuram and Keller [151]. These authors modify the object function in (1) - additively considering a second term to $u_{i k}$ to be as large as possible to avoid trivial solutions- and relax the constraints considering the conditions of a possibilistic partition, i.e. $\sum_{k=1}^{c} u_{i k}>0,0 \leq u_{i k} \leq 1$.

Barni et al., [31] highlighted some limitations in the use of the PkM clustering method proposed by Krishnapuram and Keller [152], in that these may lead to trivial solutions consisting of "coincident clusters" . This tendency may be dealt with by means of an appropriate initialization of the computational procedure (see [63]). In attempt to solve this potential problem, Krishnapuram and Keller [152] suggest an alternative objective function of the previous method.

A possibilistic clustering method based on a robust approach using Vapnik's $\varepsilon$-intensive estimator [295] has been proposed by Łęski [160].

A modification of the objective function of the first method proposed by Krishnapuram and Keller [151] has been suggested also by Yang and $\mathrm{Wu}$ [322]. Other prototype-based possibilistic clustering methods have been developed by Barni and Gualtieri [30], Ménard et al. [194] and Xie et al. [358]). Recently, possibilistic clustering methods have been proposed by Amirkhani et al. [10], Ammar et al. [11], Chang et al. [49], Maciel et al. [181], Xenaki et al. [311] and Wang and Hung [303].

Following a relational approach, possibilistic clustering methods have been proposed by Krishnapuram et al. [150] and De Càceres et al. [84].

By considering a hybrid approach, in which membership and typicality degrees are simultaneously generated, a fuzzy possibilistic clustering method has been proposed by Pal et al. [218]. Recently, a fuzzy-possibilistic clustering based on Bayesian inference has been proposed by Abadpour [1]. Successively, Abadpour [2] suggested a Bayesian inference-based fuzzy possibilistic clustering in a spatial context. Other hybrid methods have been suggested by Zhang and Leung [336] and Wu et al. [310], Aparajeeta et al. [16], Askari et al. [20] and Kannan et al. [140].

In all the mentioned possibilistic clustering methods the uncertainty is measured, in a point manner, by means of the degree of possibility (or degree of compatibility); for the hybrid methods, the uncertainty measures are, simultaneously, the membership and typicality degrees .

Note that, also in the possibilistic domain there are clustering methods in which the empirical information is affected by uncertainty, i.e. the data are imprecise. An example of possibilistic method in which the imprecise data are suitably managed has been proposed by Coppi et al. [63].

\subsection{Shadowed clustering}

Shadowed clustering is based on the concept of shadowed sets [225,229], which are defined over fuzzy sets. The main difference is that the shadowed set divides the precise numeric (point) membership values into three zones: exclusion, shadowed, and core zones (see Fig. 3). They are determined by thresholds $\lambda_{k} \in[0 ; 0.5)$. In particular, the values of the membership degrees are modified as follows $(i=1 \ldots, n ; k=1, \ldots, c)$ :

$$
u_{i k}= \begin{cases}1 & u_{i k} \geq 1-\lambda_{k} \\ u_{i k} & \lambda_{k}<u_{i k}<1-\lambda_{k} \\ 0 & u_{i k} \leq \lambda_{k} .\end{cases}
$$


In other words, the shadowed set is a modified fuzzy set. The three zones are obtained by enhancing the membership values to 1 in core zone, reducing the membership values to 0 in exclusion zone, and keeping the membership values unchanged in the shadow zone.

At the first sight shadowed sets seem to be identical to rough sets (see Section 4.4). As remarked Peters et al. [238], this is only true with respect to the categorization of the objects to three classes: (1) sure members, (2) sure non-members, and (3) objects in-between. A closer look reveals distinct differences between shadowed and rough sets, i.e. Peters et al. [238]:

- in contrast to rough sets the equivalence classes are defined dynamically in shadowed sets, i.e. the respective thresholds are derived from the data sets, whereas in rough sets the thresholds are determined by a user;

- while in rough sets that an object must belong to a least two shadowed regions (rough sets: upper approximations) if it is not a member of any core (rough sets: lower approximation) this is not true for shadowed sets.

With respect to contributes in cluster analysis, in the first paper on shadowed sets, Pedrycz [225] introduced the new concept of shadowed sets and its use in a clustering framework. Successively interesting developments concerning the utilization of shadowed sets in cluster analysis have been proposed by Pedrycz [226], in which the author suggests an interpretation of clusters in the framework of shadowed sets, and Mitra et al. [204], in which a shadowed c-means clustering is suggested integrating fuzzy and rough clustering. In particular, in the proposed method, "the elements corresponding to the shadowed region lie in the zone of uncertainty, and are treated as in FCM. However, the members of the exclusion region are incorporated in a slightly different manner. Here the fuzzy weight factor for the exclusion is designed to have the fuzzifier raised to itself, in the form of a double exponential" [204]. By taking into account these aspects, the centroids are then suitably determined. "This arrangement causes a much wider dispersion and a very low bias factor for elements which can generally be considered outside the class under discussion or most definitely, the exclusion members. This prevents the mean from getting drifted from its true value. It also minimizes the effect of noise and outliers. The threshold to induce the core, shadowed and exclusion region is automatically calculated through a functional optimization [...]. The mean [of the mathematical formula of the centroids] basically tries to first get a coarse idea regarding the cluster prototype [...] and then proceeds to tune and refine this value using data from the shadowed and exclusion region. This enables a better estimation of the actual cluster prototypes" [204]. For more details, see Mitra et al. [204].

Zhou et al. [135] proposed a shadowed set-based rough-fuzzy clustering. Mitra and Kundu [202] used the shadowed c-means clustering for satellite image segmentation. Wang and Wang [302] suggested a feature weighting fuzzy clustering integrating rough sets and shadowed sets. Zhang et al. [337] proposed a clustering method based on spatial shadowed fuzzy c-means clustering and I-Ching Operators. See, also Mitra et al. [205], Pedrycz [227], Peters et al. [238], Shi et al. [271] and Zhang et al. [337].

Within an Informational-Uncertainty perspective, the shadowed sets-based clustering methods are able to manage the uncertainty related to interpretation of the results. In particular, the shadowed sets are used to interpret the fuzzy cluster partition and distinguish between cluster cores and cluster shadows with ambiguously assigned data point.

\subsection{Rough set-based clustering}

In Section 4.1 we have illustrated the fuzzy approach to cluster analysis. Note that, in some cases, the fuzzy degree of membership may be too descriptive for interpreting clustering results [169]. Rough set-based clustering provides a solution that is less restrictive than standard clustering and less descriptive (specific) than fuzzy clustering [169].

Rough set theory has made substantial progress as a classification tool in Statistical Reasoning. The basic concept of representing a set as lower and upper approximations can be used in a broader context such as clustering. Clustering in relation to rough set theory is attracting increasing interest among researchers [169].

The notion of rough sets was proposed by Polish computer scientist Pawlak [221,222]. More formal properties and boundaries of rough sets can be found in Pawlak [222] and subsequent works. The initial and basic theory of rough sets is sometimes referred to as "Pawlak Rough Sets" or "classical rough sets", as a means to distinguish from more recent extensions and generalizations.

The theory of rough sets has emerged as another mathematical tool to manage uncertainty that arises from granularity in the domain of discourse--that is, from the indiscernibility between objects in a set. "The intention is to approximate a rough (imprecise) concept in the domain of discourse by a pair of exact concepts, called the lower and upper approximations. These exact concepts are determined by an indiscernibility relation on the domain, which, in turn, may be induced by a given set of attributes ascribed to the objects of the domain. The lower approximation is the set of objects definitely belonging to the vague concept, whereas the upper approximation is the set of objects possibly belonging to the same" [199]. A scheme of a rough set is illustrated in Fig. 4.

Since the development of rough sets, extensions and generalizations have continued to evolve. Initial developments focused on similarities and differences with fuzzy sets. While some literature argues that these concepts are different, other literature considers rough sets as a generalization of fuzzy sets - as represented through either fuzzy rough sets or rough fuzzy sets. Pawlak et al. [223] maintain that fuzzy and rough sets should be treated as being complementary to each other, addressing different aspects of uncertainty and vagueness.

In the literature, several generalizations of rough sets have been introduced, e.g. some of these generalizations are: 


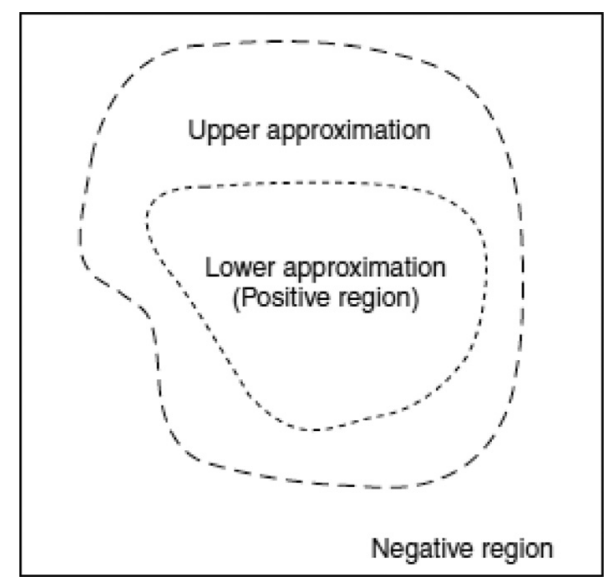

Fig. 4. Rough set approximations (i.e. lower and upper approximations) (source: [169]).

- rough multisets [117];

- fuzzy rough sets extend the rough set concept through the use of fuzzy equivalence classes [212];

- alpha rough set theory ( $\alpha$-RST) - a generalization of rough set theory that allows approximation using of fuzzy concepts [246];

- intuitionistic fuzzy rough sets [65];

- generalized rough fuzzy sets [106];

- rough intuitionistic fuzzy sets [283];

- soft rough fuzzy sets and soft fuzzy rough sets [196];

- composite rough sets [335].

An interesting discussion on rough fuzzy sets and fuzzy rough sets is shown in Dubois and Prade [102].

With respect to specific statistical area of cluster analysis, in recent years, rough sets theory has been fruitfully utilized. The first contributes on rough set-based clustering have been suggested by Lingras and West [170,171], do Prado et al. [100] and Voges et al. [299,300].

In rough clustering each cluster has two approximations, a lower and an upper approximation. The lower approximation is a subset of the upper approximation. The members of the lower approximation belong certainly to the cluster, therefore they cannot belong to any other cluster. The data objects in an upper approximation may belong to the cluster. Since their membership is uncertain they must be a member of an upper approximation of at least another cluster [236]. In particular, Lingras and West $[170,171]$ introduced a clustering method called rough c-means (RCM), which describes a cluster by a prototype (center) and a pair of lower and upper approximations. The lower and upper approximations are different weighted parameters that are used to compute the new centers.

A refined rough c-means clustering has been proposed by Peters [236].

Note that there is a crucial difference with fuzzy set theory where we also have overlapping clusters: in fuzzy set theory, an object can belong to many sets; in rough sets the memberships to two or more sets indicate that there is insufficient information to determine the actual membership to one and only one cluster [236]. See, i.e., Dubois and Prade [102], for more details.

In Fig. 5, we report some graphics drawn by Peters et al. [238], in which examples are illustrated of the differences between traditional c-means, fuzzy c-means and rough c-means in term of membership degrees. For more details, see Peters et al. [238].

In this connection, a combination of fuzzy and rough sets provides a promising approach, insofar as the two procedures complement each other to some extent. In fact, various clustering methods combining rough and fuzzy sets have been proposed in the last decade.

In particular, we focus our attention on two rough-fuzzy clustering methods which, as remarked by Peters et al. [238], while named identically, integrate fuzzy concepts into the rough c-means in slightly differently ways:

- Mitra et al.'s Rough-Fuzzy Clustering [206]: The authors proposed a hybrid rough-fuzzy clustering algorithm (RFCM) with fuzzy lower approximations and fuzzy boundaries. The RFCM mainly differs from the original rough clustering method (RCM) [238] in the following:

(i) with respect to the assignment of the objects to a lower approximation or a boundary: Euclidean distances used in rough c-means to distinguish between the objects in the boundary and in the lower approximations are replaced with membership degrees obtained by fuzzy clustering;

(ii) with respect to the calculation of the means: when calculating the means in rough clustering, the objects in the lower approximation as well as in the boundary are weighted based on their respective fuzzy membership degrees. 

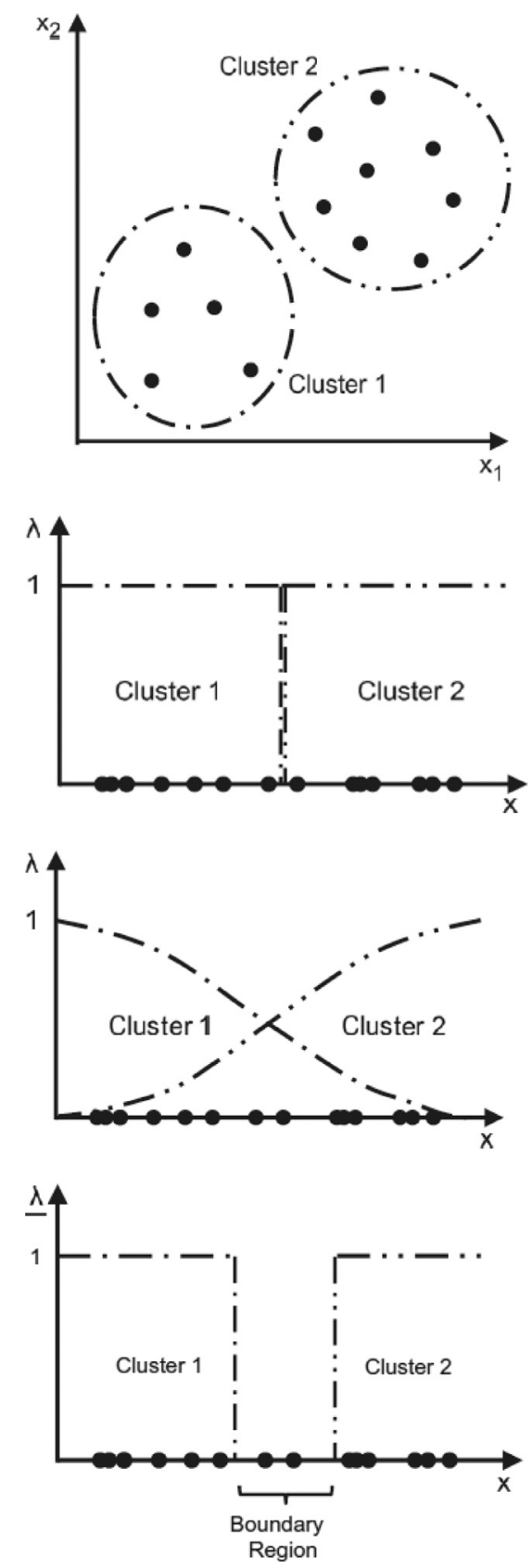

Fig. 5. Membership degrees for traditional c-means, fuzzy c-means and rough c-means in terms of membership degrees (source: [238]) (we consider the number of clusters of the original paper, i.e. two clusters; furthermore, we consider the original notation of the membership degree, i.e., $\lambda$ ). In Figure, we show, respectively, the data pattern (grouping objects in clusters, the membership degrees for traditional, fuzzy and rough c-means clustering.

- Maji and Pal's Rough-Fuzzy Clustering [183]: the authors propose a variant of Mitra et al.'s [206] hybrid rough-fuzzy clustering algorithm. All objects in a lower approximation have the same influence on the determination of their means and are independent from other clusters. Hence, they propose a rough-fuzzy c-means with crisp lower approximations and fuzzy boundaries. Obviously, in Maji and Pal's [184] method the lower approximation has a higher impact on clustering in comparison to Mitra et al.'s [206] approach where the objects in lower approximation are weighted by factors between 0 and 1 [238].

In addition, Maji and Pal [184] also proposed a rough set -based generalized fuzzy c-means algorithm which they called RFPCM (rough fuzzy possibilistic c-means). The algorithm merges fuzzy and possibilistic approaches and rough k-means in a way that the three clustering algorithms it includes can be derived from it. As in Maji and Pal's [183] RFCM, a cluster 


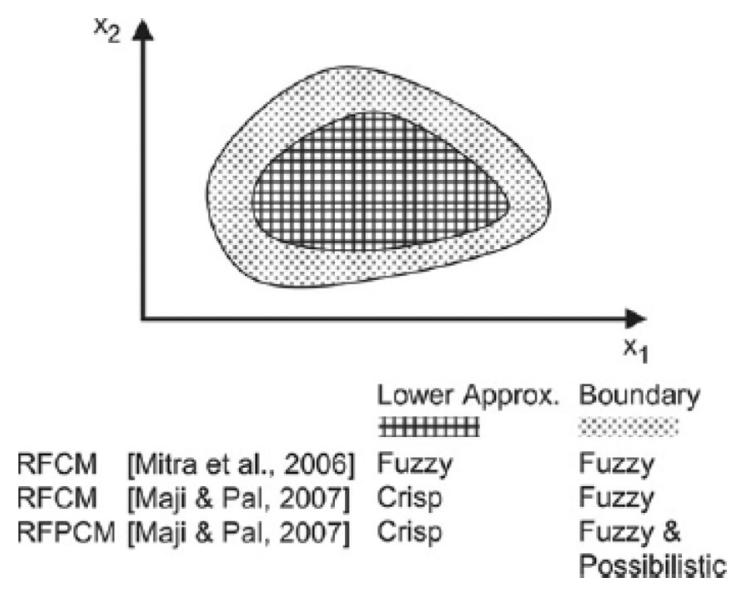

Fig. 6. Scheme of Mitra et al.'s [206], Maji and Pal's [183] and Maji and Pal [184] methods (source: [238]).

is approximated by a crisp lower approximation and a fuzzy boundary. Additionally, in their RFPCM, the boundary can be characterized by possibilistic elements.

In Fig. 6, we show the original graphic drawn by Peters et al. [238], which summarizes Mitra et al.'s [206] and Maji and Pal's [183] versions of the rough-fuzzy c-means clustering, and rough fuzzy possibilistic c-means clustering proposed by Maji and Pal [184].

In Mitra et al. [206], the centroids are calculated based on the weighting average of the fuzzy lower approximation and fuzzy boundary. In Maji and Pal [183] and Maji and Pal [184], the centroids are calculated based on the weighting average of the crisp lower approximation and fuzzy boundary. Computation of the centroids is modified to include the effects of both fuzzy memberships -i.e. fuzzy memberships for the first method, fuzzy and possibilistic memberships for the second oneand lower and upper bounds.

Within the Informational Paradigm perspective, as in the case of the previous rough-fuzzy clustering methods, the uncertainty affecting the information is managed by means of the concepts of lower and upper approximations and fuzzy boundary.

An interesting description of the basic concept of rough clustering based on k-means, genetic algorithms, Kohonen selforganizing maps, and support vector clustering is found in Lingras and Peters [169]. Their article also includes a review of rough cluster validity measures, and applications of rough clustering to such diverse substantive areas.

Other clustering methods based on rough sets have been proposed recently by Cai and Verbeek [43], Hamidzadeh et al. [123], Shi et al. [271] and Pacheco et al. [217].

For a survey of rough clustering and its extensions and derivatives see also Peters et al. [238].

References relative to several contributes on rough clustering, extensions and derivatives suggested in the literature are listed chronologically in Table 2.

\subsection{Intuitionistic fuzzy clustering}

As mentioned above, since Zadeh introduced fuzzy sets in 1965, various approaches and theories treating imprecision and uncertainty have been proposed. In 1986, Atanassov [25] introduced the concept of an Intuitionistic Fuzzy Set (IFS) which is characterized by two functions expressing respectively the degree of belonging (degree of membership) and the degree of non-belonging (degree of non-membership).

Mathematically speaking, an Intuitionistic Fuzzy Set can be defined as follows.

Let $\mathrm{X}$ be an initial universe. An Intuitionistic Fuzzy Set (IFS) $\mathrm{A} \subseteq \mathrm{X}$ is defined as follows:

$$
\mathrm{A}=\left\{\left(\mathrm{x}, \mu_{\mathrm{A}}(\mathrm{x}), v_{\mathrm{A}}(\mathrm{x}): \mathrm{x} \in \mathrm{X}\right\}\right.
$$

where $\mu_{\mathrm{A}}(\mathrm{x})$ represents the degree of membership of $\mathrm{x}$ to $\mathrm{A}, v_{\mathrm{A}}(\mathrm{x})$ indicates the degree of non-membership of $\mathrm{x}$ to $\mathrm{A}$ with the constraints:

$$
\mu_{\mathrm{A}}(\mathrm{x}) \in[0,1], v_{\mathrm{A}}(\mathrm{x}) \in[0,1] \text { and } 0 \leq \mu_{\mathrm{A}}(\mathrm{x})+v_{\mathrm{A}}(\mathrm{x}) \leq 1
$$

and then $\pi_{A}(x)=1-\left(\mu_{A}(x)+v_{A}(x)\right)$ indicates the degree of hesitancy of $x$ to $A$.

As remarked by Grzegorzewski and Mròwka [116] "Atanassov's sets give us a very natural tool for modeling preferences. Sometimes it seems to be more natural to describe imprecise and uncertain opinions not only by membership functions. It is due to the fact that in some situations it is easier to describe our negative feelings than positive ones. Even more, quite often one can easily specify objects or alternatives one dislikes, but simultaneously cannot specify clearly what he really wants." For more details on Intuitionistic Fuzzy Sets see, e.g. Atanassov [21,22,25], Atanassov and Gargov [23] and Xu [314]. 
Table 2

Contributes on rough set-based clustering.

\begin{tabular}{ll}
\hline Lingras and West [170] & Xue et al. [319] \\
Questier et al. [247] & Mitra et al. [204] \\
do Prado et al. [100] & Pedrycz [230] \\
Voges et al. [300] & Maji [182] \\
Voges et al. [299] & Chen and Zhang [53] \\
Lingras et al. [172] & Zhou et al. [135] \\
Asharaf and Murty [19] & Lingras and Peters [169] \\
Mitra et al. [203] & Maji and Paul [186] \\
Lingras and West [171] & Wang and Zhou [301] \\
Kumar De and Krishna [155] & Mitra et al. [205] Maji and Paul [187] \\
Shuai-Dong and Shi-Hong [272] & Yanto et al. [323] \\
Mitra [199] & Peters et al. [242] \\
Lingras and West [171] & Nayak et al. [214] \\
Asharaf et al. [17] & Cheng and Wu [58] \\
Mitra [200] & Crespo et al. [71] \\
Peters [235] & Peters et al. [238] \\
Peters and Lampart [239] & Joshi and Lingras [136] \\
Asharaf et al. [18] & Lai et al. [158] \\
Chen and Wang [52] & Maji and Paul [188] \\
Peters [236] & Gao et al. [108] \\
Mitra et al. [206] & Chen et al. [56] \\
Kumar et al. [156] & Li et al. [161] \\
Parmar et al. [220] & Ma et al. [180] \\
Lingras [167] & Peters [237] \\
Maji and Pal's [183] & Zhang et al. [338] \\
Maji and Pal [184] & Li et al. [162] \\
Peters et al. [240] & Park and Choi [219] \\
Maji and Pal [185] & Cai and Verbeek [43] \\
Mitra and Barmann [201] & Shi et al. [271] \\
Bean and Kambhampati [32] & Hamidzadeh et al. [123] \\
Viswanath and Babu [298] & Pacheco et al. [217]. \\
Malyszko and Stepaniuk [189] & \\
Kishida [145] & \\
Herawan et al. [125] & \\
\hline & \\
\hline
\end{tabular}

In the literature, there has been a lively debate by some scholars on the appropriateness of the term intuitionistic fuzzy sets (IFS) adopted by Atanassov for his theory. Grzegorzewski and Mròwka [116] suggested a list of possible alternative terms that would however allow one to retain the acronym IFS: incomplete fuzzy sets, inaccurate fuzzy sets, imperfect fuzzy sets, indefinite fuzzy sets, indeterminate fuzzy sets, indistinct fuzzy sets. Dubois et al. [103] suggested inappropriate fuzzy sets, interval fuzzy sets, imprecise fuzzy sets. Atanassov [24] took part in the discussion replying to the observations of the scholars involved in the debate.

The theory of Intuitionistic Fuzzy Sets has been used successfully in cluster analysis for modeling uncertainty in the clustering process and various clustering methods have been proposed.

Hung et al. [129] proposed a fuzzy clustering method based on intuitionistic fuzzy tolerance relations. Pelekis et al. [234] proposed a variant of FcM that copes with uncertainty and involves a similarity measure between the intuitionistic fuzzy sets, which is appropriately integrated into the clustering procedure. Torra et al. [292] defined a clustering method to construct an intuitionistic fuzzy partition that deals with the uncertainty present in different executions of the same clustering procedure. Xu et al. [316] proposed a clustering algorithm based on the association coefficients of intuitionistic fuzzy sets. This clustering algorithm is similar to the clustering technique proposed by Hung et al. [129]. Cai et al. [44] suggest a clustering technique based on the use of the intuitionistic fuzzy dissimilarity matrix and the so-called $(\alpha, \beta)$-cutting matrices. Also this method is similar to that one proposed by Hung et al. [129]. Karthikeyani et al. [142] described an intuitionistic fuzzy approach to distributed fuzzy clustering. A simple clustering method for classifying data represented by intuitionistic fuzzy estimates has been suggested by Todorova and Vassilev [288]. Xu [313] developed an agglomerative hierarchical clustering algorithms for classifying ordinary intuitionistic fuzzy sets and interval-valued intuitionistic fuzzy sets. $\mathrm{Xu}$ and $\mathrm{Wu}$ [317] defined an intuitionistic fuzzy c -means method for intuitionistic fuzzy sets. Wang et al. [304] proposed a method for constructing an intuitionistic fuzzy tolerance matrix from a set of intuitionistic fuzzy sets and a netting method for clustering intuitionistic fuzzy sets via the corresponding intuitionistic fuzzy tolerance matrix. Zhao et al. [345] proposed an intuitionistic fuzzy minimum spanning tree clustering algorithm to deal with intuitionistic fuzzy information. Zhao et al. [346] proposed a measure for computing the association coefficient among intuitionistic fuzzy sets, an algorithm for clustering intuitionistic fuzzy sets and successively extended the algorithm to classify interval-valued intuitionistic fuzzy sets. Wang et al. [305] defined an intuitionistic fuzzy implication operator and extended the Lukasiewicz implication operator to intuitionistic fuzzy environments, and then defined an intuitionistic fuzzy triangle product and an intuitionistic fuzzy square 
Table 3

Contributes on intuitionistic fuzzy set-based clustering.

\begin{tabular}{ll}
\hline Hung et al. [129] & [126] \\
Torra et al. [292] & Thong and Son [284] \\
Pelekis et al. [234] & Verma and Agrawal [296] \\
Xu et al. [316] & Agrawal and Tripathy [3] \\
Xu [313] & Aliahmadipour and Eslami [5] \\
Cai et al. [44] & Ananthi et al. [12] \\
Karthikeyani Visalakshi et al. [142] & Balasubramaniam and Ananthi [27] \\
Todorova and Vassilev [288] & Chen and Liu [57] \\
Xu and Wu [317] & Dubey et al. [101] \\
Chaira [47] & Kacprzyk et al. [137] \\
Wang et al. [304] & Kaushik et al. [143] \\
Liu et al. [178] & Prabu et al. [244] \\
Rangasamy et al. [250] & Shang et al. [270] \\
Son et al. [280] & Tripathy et al. [293] \\
Xu [314] & Tripathy et al. [294] \\
Zhao et al. [345] & Verma et al. [297] \\
Xu et al. [312] & Zhou et al. [352] \\
Zhao et al. [346] & \\
Chaira and Panwar [48] & \\
Lin [165] & \\
Wang et al. [305] & \\
\hline
\end{tabular}

product. Moreover, they used the intuitionistic fuzzy square product to construct an intuitionistic fuzzy similarity matrix, based on which a direct method for intuitionistic fuzzy clustering has been proposed.

Recently, intuitionistic fuzzy clustering methods have been proposed by Agrawal and Tripathy, [3], Aliahmadipour and Eslami [5], Ananthi et al. [12], Chen and Liu [57], Dubey et al. [101], Kacprzyk et al. [137], Prabu et al. [244], Shang et al. [270], Tripathy et al. [293,294] and Zhou et al. [351].

A chronological list of the main contributions to intuitionistic fuzzy sets-based clustering is shown in Table 3 .

From an Information-Uncertainty perspective, note that the various contributions to intuitionistic fuzzy clustering focus on the uncertainty associated with the assigning of objects to clusters, not measured in terms of point membership degree, but by means of two uncertainty measurements, i.e. the degree of belonging (degree of membership) and the degree of non-belonging (degree of non-membership).

\subsubsection{Vague clustering}

Since fuzzy sets theory is based on the concept of point-based membership, vagueness can be described in inaccurate ways. Motivated by this, Gau and Buehrer [109] elaborated the concept of vague sets. In vague sets, the membership function value is an interval-based membership, i.e., a subinterval of $[0,1]$.

In a clustering domain, Xu et al. [312] proposed a fuzzy clustering method in the context of vague sets theory and fuzzy c-means clustering, i.e., a vague c-means clustering method (VcM). In the objective function of VcM, membership degrees are not point-based memberships but interval-based memberships based on the so-called truth-memberships and falsememberships. Note that, as pointed out by Bustince and Burillp [40], vague sets are equivalent to intuitionistic fuzzy sets; for this reason, the vague c-means clustering method suggested by Xu et al. [312] can be considered an intuitionistic fuzzy clustering.

\subsection{Evidential clustering or credal clustering or Belief clustering}

The theory of belief functions, also referred to as evidence theory or Dempster-Shafer theory (DST), is a general framework for reasoning with uncertainty, related to probability, possibility and imprecise probability theories. First introduced by Arthur P. Dempster [90] in the context of statistical inference, the theory was later developed by Glenn Shafer [268] into a general framework for modeling epistemic uncertainty-a mathematical theory of Evidence. The theory makes it possible to combine evidence from different sources and arrive at a degree of belief (represented by a mathematical object called belief function) that takes into account all the available evidence. Dempster-Shafer theory is a generalization of the Bayesian probability Bayesian theory of subjective probability. Belief functions base degrees of belief (or confidence, or trust) for one question on the probabilities for a related question. The degrees of belief itself may or may not have the mathematical properties of probabilities; how much they differ depends on how closely the two questions are related. Dempster-Shafer's theory is based on two principles: obtaining degrees of belief for one question from subjective probabilities for a related question, and Dempster's rule for combining such degrees of belief when they are based on independent items of evidence. In essence, the degree of belief in a proposition depends primarily upon the number of answers (to the related questions) containing the proposition, and the subjective probability of each answer. Also contributing are the rules of combination that reflect general assumptions about the data. In this formalism, a degree of belief (also referred to as a mass) is represented as a belief function rather than a Probability distribution. Probability values are assigned to sets of possibilities rather 
than single events: their appeal rests on the fact they naturally encode evidence in favor of propositions. Dempster-Shafer theory assigns its masses to all of the non-empty subsets of the propositions that compose a system-in Set Theory terms, the Power Sets of the propositions.

Successively, Cozman [68-70] proposed the so-called Credal Set (CS) theory, which is a generalization of Bayesian theory, in which one acknowledges that there might be more than one reasonable function to represent belief and evidence. Thus, CS theory is strongly associated with theory of evidence [90,268].

For more details in Dempster-Shafer theory and Credal Set (CS) theory see, respectively, Dempster, [90], Shafer [268] and Cozman [68-70]. In the last decade, the credal theory and the Dempster-Shafer theory -due to their strong theoretical and methodological potential- have had a great success in Statistical Reasoning, especially thanks to the French school headed by Denœux and Masson who produced several relevant theoretical results above all in the field of cluster analysis. The clustering methods based on the credal theory and the Dempster-Shafer theory are often indicated in the literature by different terms with the same meaning, such as Evidential clustering, Belief clustering, Dempster-Shafer clustering and Credal clustering.

Credal clustering (or evidential clustering) represents the uncertainty about the membership of objects to clusters using the formalism of belief functions. In particular, in a credal partition, the membership of each object to clusters is described by a mass function, i.e., a function that assigns a mass between 0 and 1 to each set of clusters, with the constraint that the masses sum to 1 .

Paraphrasing the same authors, the first incursion of belief function into cluster analysis domain it was carried out by Denœux and Masson in 2004. In fact, Denœux and Masson [97] proposed a novel approach to clustering proximity data, based on the belief functions, called evidential clustering (EVCLUS). In their clustering method, the allocation of objects to classes is performed using the concept of basic belief assignment (bba), whereby a "mass of belief" is assigned to each possible subset of classes. Having assigned a bba to each object, it is possible to compute, for each two objects, the plausibility that they belong to the same class. It is then required that these plausibilities be, in some sense, compatible with the observed pairwise dissimilarities between objects [97]. "As the concept of fuzzy partition subsumes that of crisp partition, resulting in greater expressive power of fuzzy clustering procedures as compared with hard ones, the concept of credal partition introduced [in EVCLUS] is even more general, which allows in some cases to gain deeper insight into the structure of the data. Additionally, evidential clustering provides the possibility to combine in a meaningful way credal partitions obtained from dissimilarity matrices provided, e.g., by several experts, or computed from different sets of measurements" [97]. In the same year, Masson and Denœux [97] suggested a new version of EVCLUS for interval-valued proximity data. Successively, the same authors [190] proposed an evidential version of the fuzzy c-means clustering, i.e. evidential c-means (ECM) in which they address the problem of computing a credal partition from object data. Masson and Denœux [191] also defined a relational evidential c-means algorithm (RECM) for proximity data. In 2011, Masson and Denœux [192] investigated, in the belief functions framework (defined on the lattice of intervals partitions of a set of objects), the problem of finding a consensus clustering as an information fusion problem in the general framework of uncertain reasoning. Antoine et al. [14] suggested to introduce pairwise constraints in the ECM method in order to define the so-called constrained evidential c-means algorithm (CECM), which combines the advantages of adding background knowledge and using belief functions. Denœux and Masson [98] discussed the Evidential reasoning in large partially ordered sets with application to multi-label classification, ensemble clustering and preference aggregation. Liu et al. [177] proposed a new Belief C-Means (BCM) algorithm, in which the mass of belief of the specific cluster for each object is computed from the distance between the object and the center of the cluster, and the mass of belief of a meta-cluster is computed both from the distances between object and prototypes of the involved specific clusters, and the distances among these prototypes. In BCM, there is no need to compute the barycenter of the meta-clusters. Serir et al. [267] suggested an evidential evolving Gustafson-Kessel algorithm for online data streams partitioning using belief function theory. Antoine et al. [15] proposed an evidential clustering with instance-level constraints for relational data (CEVCLUS). Denœux et al. [96] introduced a new decision-directed evidential clustering algorithm (EK-NNclus) based on the EK-NN rule [92]. Zhou et al. [348] extended the median clustering methods in the framework of belief functions theory and put forward the Median Evidential C-Means (MECM) algorithm. By using a new concept of meta-clusters, Liu et al. [179] proposed an evidential version of FCM called credal c-means (CCM) to overcome the limitation of ECM. CCM method differs from BCM method because it is based on a distinct underlying principle and a different interpretation of the meta-clusters. CCM also works with credal partition for the clustering of imprecise data based on belief functions. Guo and Şengür [120] suggested a neutrosophic (see Section 4.9) evidential c-means (NECM). Denœux et al. [95] proposed some improvements to the EVCLUS algorithm, making it applicable to very large datasets. Zhou et al. [351] introduced the so-called ECMdd method, i.e. Evidential c-medoids clustering method with multiple prototypes. For other recent papers on evidential clustering, see Denoeux and Kanjanatarakul [93,94], Kanjanatarakul et al. [139], Li and Wang [164].

Recently, various algorithms introduced by the French school headed by Denœux and Masson -i.e. Evidential cMeans (ECM), Relational Evidential c-Means (RECM), Constrained Evidential c-Means (CECM), EVCLUS and EK-NNclus- have been implemented in the $\mathrm{R}$ software "evclust: Evidential Clustering', [91]. For more details, see the link: https://cran.r-project.org/web/packages/evclust/vignettes/Introduction.html

The various contributes on credal clustering (evidential clustering) are shown chronologically in Table 4. 
Table 4

Contributes on credal clustering (evidential clustering).

\begin{tabular}{ll}
\hline Denœux and Masson [97] & Zhou et al. [350] \\
Masson and Denœux [190] & Liu et al. [179] \\
Masson and Denœux [191] & Guo and Şengür [120] \\
Masson and Denœux [192] & Denœux [91] \\
Antoine et al. [14] & Denoeux and Kanjanatarakul [93] \\
Denœux and Masson [98] & Denœux et al. [95] \\
Liu et al. [178] & Kanjanatarakul et al. [139] \\
Serir et al. [267] & Li and Wang [164] \\
Antoine et al. [15] & Zhou et al. [351] \\
Denœux et al. [96] & Denoeux and Kanjanatarakul [94]. \\
\hline
\end{tabular}

Note that the various clustering algorithms previously illustrated produce a credal partition, i.e., a set of Dempster-Shafer mass functions representing the membership of objects to clusters. The mass functions quantify the cluster-membership uncertainty of the objects.

\subsection{Credibilistic clustering}

Fuzzy set theory has been extensively developed and applied to a wide variety of real-life problems. Among these developments are the possibility theory and the credibility theory, respectively based on the measure of possibility and of credibility.

Although possibility measures are widely used, they are not self-dual. And, as remarked by Liu and Liu [173], self-dual measures are absolutely needed in both theory and practice. To address this issue, Liu and Liu proposed the use of credibility measures in 2002 [176], which avoid this issue, and are similar to probability measures. From then on, credibility measures have been widely applied to definitions, e.g., of expected value [176] or credibility distribution [173]. Li and Liu [163] also studied the relation between possibility measures and credibility measures, and demonstrated a sufficient and necessary condition for credibility measures. They showed that, compared to possibility measures, the advantage of credibility measures is their self-dual property. Their paper shows a relation between possibility measures and credibility measures, and demonstrates a sufficient and necessary condition for credibility measures.

Generally speaking, credibility theory is the branch of mathematics that studies the behavior of fuzzy events. A detailed survey of credibility theory may be found in Liu [175]. An axiomatic foundation for credibility theory was provided by Liu in 2004 [174].

Recently, credibilistic theory has had a positive impact in some areas of Statistical Reasoning, such as cluster analysis. Zhou et al. [347] proposed a clustering method based on FCM. They applied credibility weights to measure the compactness of data, but they did not apply them to minimize the objective function because of predefined membership functions. Their method has been used by Wen et al. [307] as a basis for an image segmentation algorithm. Zhou et al. [349] applied a hybrid method of spatial credibilistic clustering [347] and particle swarm optimization (SCCPSO). Wen et al. [308] suggested a modified SCCPSO. Niakan et al. [216] proposed a new credibilistic clustering method is introduced in which credibility measure is applied instead of possibility measure in possibilistic clustering. In Kalhori and Zarandi [138], the credibility theory is used with a different approach. "In particular, the credibility of an event, as the average of possibility and necessity of it, is pointed out. So, the possibility of the event, membership of a data in a cluster, and its necessity are calculated from objective function. Then, the credibility of this event is calculated using average of possibility and necessity. The possibility of membership of a data in a cluster is gained considering the distance of it from the other cluster centers; on the other hand, the possibility of non-membership of this data in the cluster is gained regarding the effect of membership of this data in the cluster on extension of its borders and decrement of its separation from the other clusters. The necessity of the event membership of the data in a cluster is complement of the possibility of event non-membership. After calculating the possibility and necessity of this event, its credibility is calculated as the average of its possibility and necessity" [138]. Note that, the proposed objective function of method suggested by Kalhori and Zarandi is interval type-2 (see Section 4.8) based on credibility measures; for this reason, the method is called Interval Type-2 Credibilistic Clustering (IT2CC).

To solve the "coincident problem" affecting the possibilistic clustering, Zhou et al. [348] suggest a credibilistic clustering method. In the proposed clustering algorithm, the objective function is the compactness index of the data sets with credibilistic membership weights, and the constraints on credibilities are deduced from the mathematical properties of the credibility measure. Furthermore, in order to solve this model with good clustering results, a credibilistic clustering algorithm based on the alternating cluster estimation method. For more, details, see Zhou et al. [348].

In line with the Informational-Uncertainty framework, uncertainty is measured in this case by means of a credibilistic measure.

\subsection{Type-2 fuzzy clustering}

Type-2 Fuzzy Set (T2FS) was introduced by Zadeh [353] as an extension of the concept of an ordinary Fuzzy Set (or Type1 Fuzzy Set) and is essentially "fuzzy fuzzy" sets where the fuzzy degree of membership is a type-1 fuzzy set. Successively, it was investigated by Mizumoto and Tanaka [208,209]. 

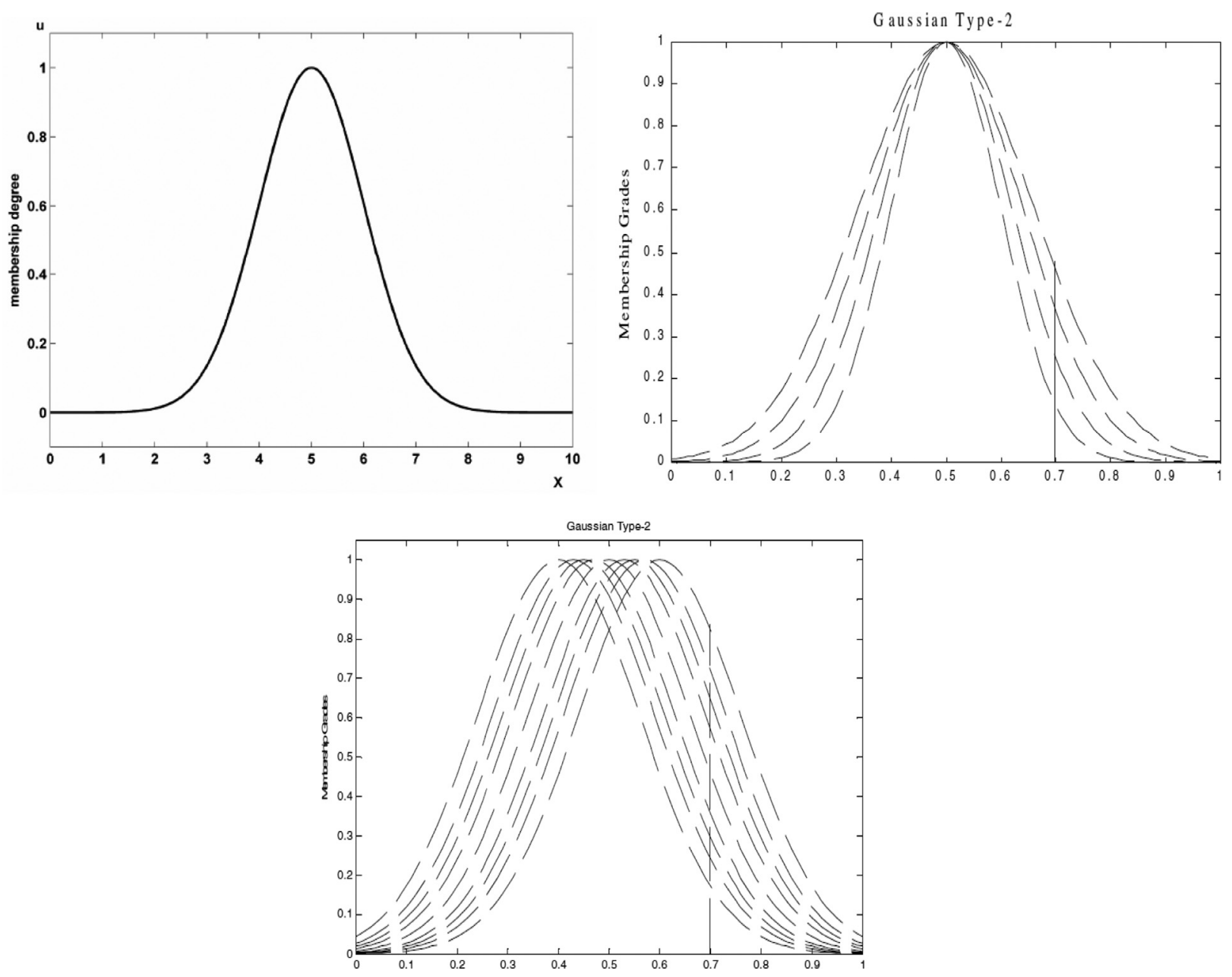

Fig. 7. Gaussian membership function for a Type-1 fuzzy set, for a Type-2 fuzzy set representing a type-1 fuzzy set with uncertain standard deviation and for a Type-2 fuzzy set representing a type-1 fuzzy set with uncertain mean (the mean is uncertain in the interval [0.4, 0.6]) (source: [46]).

A Type-2 Fuzzy Set (T2FS) is characterized by a fuzzy membership function, i.e., the membership grade for each element of this set is a fuzzy set in [0,1], unlike a type-1 set where the membership grade is a crisp number in [0,1]. Such set can be used in situations where there is uncertainty about the membership grades themselves, e.g., an uncertainty in the shape of the membership function or in some of its parameters [46].

When we cannot determine the membership of an element in a set as 0 or 1 , we use Type-1 Fuzzy Sets; when the situation is so fuzzy that we have trouble determining membership grade even as a crisp number in [0,1], we use Type-2 Fuzzy Sets. This does not mean that we need to have extremely fuzzy situations to use Type-1 Fuzzy Sets. Type- 1 Fuzzy Sets can be considered as a first order approximation to real-world uncertainty and Type-2 Fuzzy Sets as a second order approximation [46].

It is possible to consider fuzzy sets of higher types but the complexity of the fuzzy system increases very rapidly. For this reason, we will only focus on Type-2 Fuzzy Sets.

In Fig. 7, we show an example of Gaussian membership function for a Type-1 fuzzy set (Fig. 7a), for a Type-2 fuzzy set representing a type- 1 fuzzy set with uncertain standard deviation (Fig. 7b) and for a Type-2 fuzzy set representing a type- 1 fuzzy set with uncertain mean (the mean is uncertain in the interval [0.4, 0.6]) (Fig. 7c) drawn by Castillo and Melin [46].

In Fig. 8, we consider a Type-1 fuzzy set characterized by a Gaussian membership function (mean $\mathrm{M}$ and standard deviation $\left.\sigma_{\mathrm{x}}\right)$, which gives one crisp membership $\mathrm{m}(\mathrm{x})$ for each input $\mathrm{x} \in \mathrm{X}$, where

$$
\mathrm{m}(\mathrm{x})=\exp \left\{-1 / 2\left[(\mathrm{x}-\mathrm{M}) / \sigma_{\mathrm{x}}\right]^{2}\right\} .
$$

Now, imagine that this membership of $\mathrm{x}$ is a fuzzy set. Let us call the domain elements of this set "primary memberships" of $\mathrm{x}$ (denoted by $\mu_{1}$ ) and membership grades of these primary memberships "secondary memberships" of $\mathrm{x}$ [denoted by $\left.\mu_{2}\left(\mathrm{x}, \mu_{1}\right)\right]$. So, for a fixed $\mathrm{x}$, we get a Type- 1 fuzzy set whose domain elements are primary memberships of $\mathrm{x}$ and whose corresponding membership grades are secondary memberships of $\mathrm{x}$. If we assume that the secondary memberships follow 


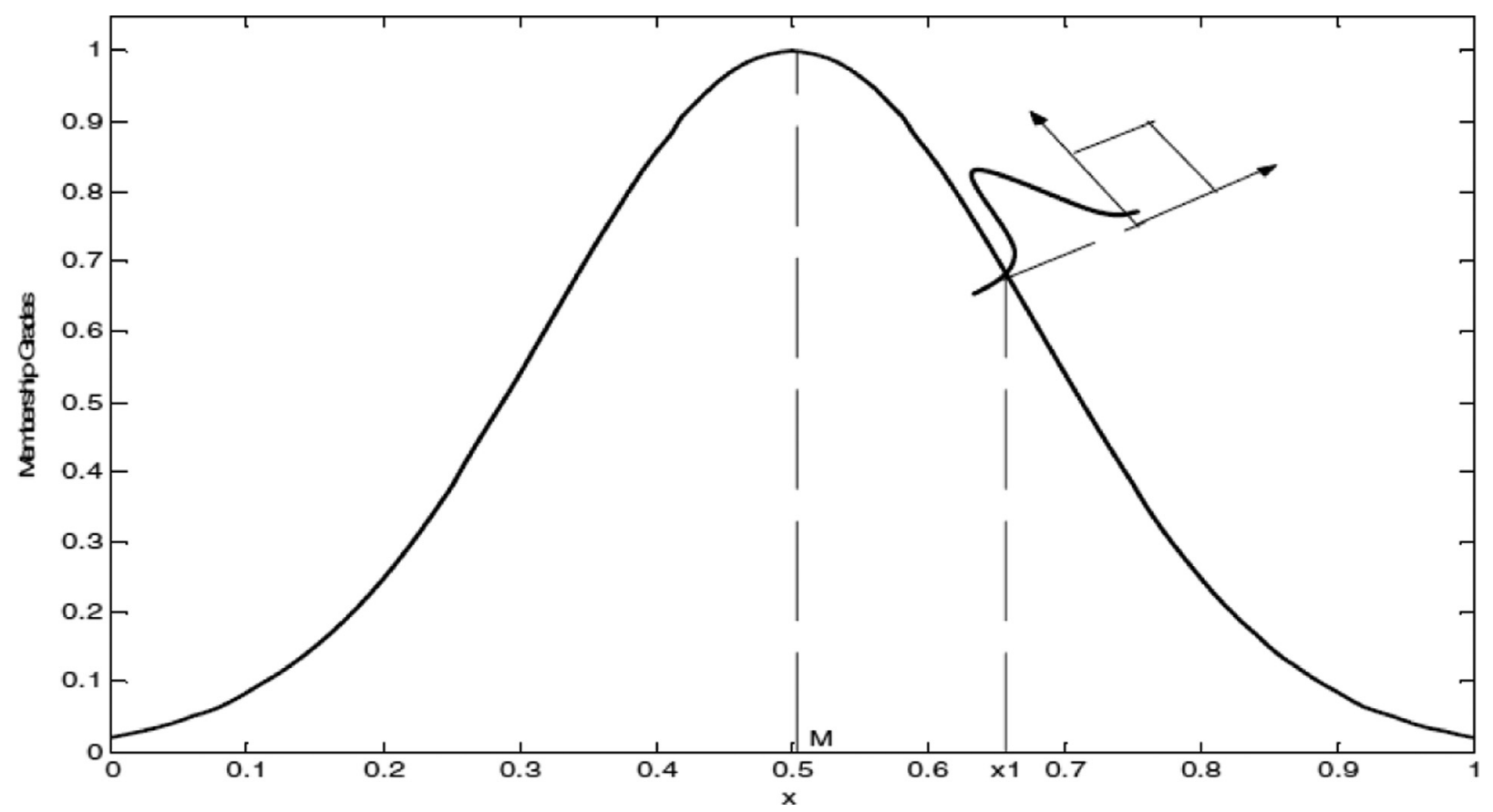

Fig. 8. A Type-2 fuzzy set in which the membership grade of every domain point is a Gaussian Type-1 fuzzy set (source: [46]).

a Gaussian with mean $\mathrm{m}(\mathrm{x})$ and standard deviation $\sigma_{\mathrm{m}}$, as in Fig. 8, we can describe the secondary membership function for each $\mathrm{x}$ as

$$
\mu_{2}\left(\mathrm{x}, \mu_{1}\right)=\exp \left\{-1 / 2\left[\left(\mu_{1}-\mathrm{m}(\mathrm{x})\right) / \sigma_{\mathrm{m}}\right]^{2}\right\}
$$

where $\mu_{1} \in[0,1]$ and $\mathrm{m}$ is as in Eq. (1) [46].

We can define the two more used kinds of Type-2 fuzzy sets as follows [46]:

- a Gaussian Type-2 Fuzzy Set (GaT2FS) is one in which the membership grade of every domain point is a Gaussian type-1 set contained in $[0,1]$;

- an Interval Type-2 Fuzzy Set (IT2FS) is one in which the membership grade of every domain point is a crisp set whose domain is some interval contained in $[0,1]$.

For remarking the general definition of T2FS with respect to particular cases, such as GaT2FS and IT2FS, in the literature it is often used for T2FS the acronym GT2FS (General Type-2 Fuzzy Set).

We observe that uncertainty in the primary memberships of a type-2 fuzzy set, A, consists of a bounded region that we call the footprint of uncertainty (FOU). Mathematically, it is the union of all primary membership functions [195].

An upper membership function and a lower membership functions are two Type-1 membership functions that are bounds for the FOU of a Type- 2 fuzzy set A. The upper membership function is associated with the upper bound of FOU(A). The lower membership function is associated with the lower bound of $\mathrm{FOU}(\mathrm{A})$.

In Fig. 9 (drawn by [46]) we illustrate the concept of upper and lower membership functions as well as the footprint of uncertainty for a Type-2 Gaussian membership function. This footprint of uncertainty can be obtained by projecting in two dimensions the three-dimensional view of the Type-2 Gaussian membership function.

Mathematically speaking, in general, a Type-2 Fuzzy Sets (T2FS) can be defined in the following way.

Let $\mathrm{X}$ be an initial universe. A Type-2 Fuzzy Set (T2FS) $\mathrm{A} \subseteq \mathrm{X}$ is defined as follows:

$$
\mathrm{A}=\left\{\left(\mathrm{x}, \mathrm{y}, \xi_{\mathrm{A}}(\mathrm{x}, \mathrm{u}): \mathrm{x} \in \mathrm{X}, \mathrm{u} \in[0,1]\right\},\right.
$$

where $\xi_{A}(x, u)$ represents the degree of membership function of $(x, u)$ to $A, x \in X, u \in[0,1]$.

Often, it is more convenient to write $\xi_{\mathrm{A}}(\mathrm{x}, \mathrm{u})$ as the product $\mu_{\mathrm{A}}(\mathrm{x}, \mathrm{u}) \mathrm{v}_{\mathrm{A}}(\mathrm{x}, \mathrm{u})$, where

$$
\begin{aligned}
& \mu_{A}(x)=\max _{y}\left(\xi_{A}(x, u)\right), \\
& v_{A}(x, u)=\left\{\begin{array}{lll}
\frac{\xi_{A}(x, u)}{\mu_{A}(x)} & \text { if } & \mu_{A}(x)>0 \\
1 & \text { otherwise }
\end{array}\right.
\end{aligned}
$$

are known, respectively, as the primary and the secondary membership functions [197]. 


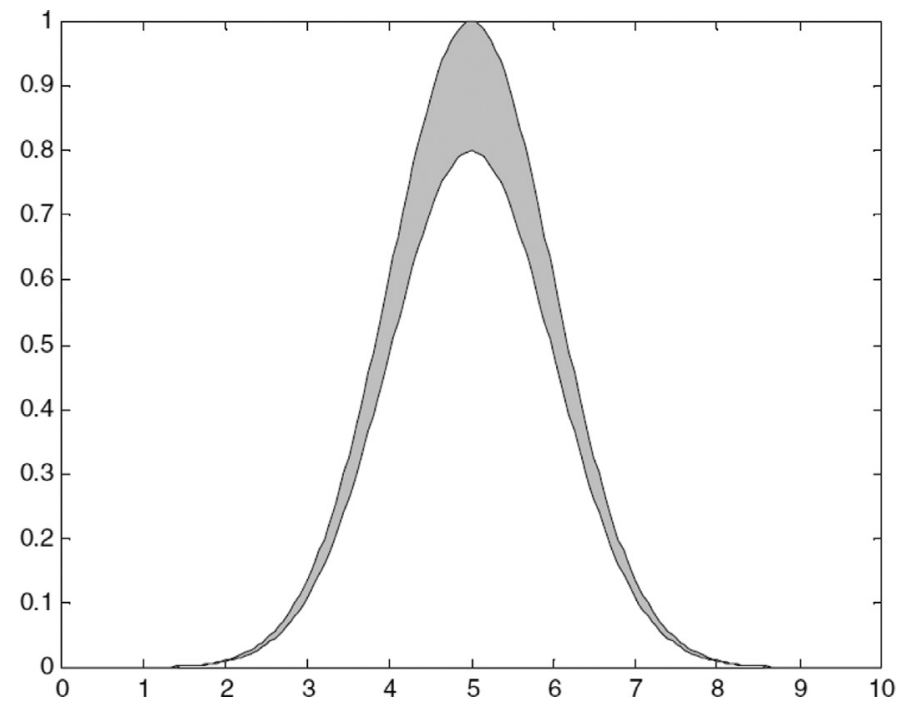

Fig. 9. Upper and lower membership functions and footprint of uncertainty for a Type-2 Gaussian membership function (source: [46]).

We delineate the region $\xi_{\mathrm{A}}(\mathrm{x}, \mathrm{u})>0$, by means of the a low membership function $\psi_{\mathrm{A}}(\mathrm{x})$, and a high membership function $\phi_{\mathrm{A}}(\mathrm{x})$, where

$$
\xi_{\mathrm{A}}(\mathrm{x}, \mathrm{u})>0 \text { if } \psi_{\mathrm{A}}(\mathrm{x}) \leq \mathrm{u} \leq \phi_{\mathrm{A}}(\mathrm{x}) .
$$

Physically, the difference $\left|\phi_{A}(x)-\psi_{A}(x)\right|$ represents the uncertainty in specifying the primary membership value $\mu_{A}(x)$ [197].

Thus, from the definition of T2FS, we can obtain the definition of IT2FS as follow:

Let $\mathrm{X}$ be an initial universe. An Interval Type-2 Fuzzy Set (T2FS) A $\subseteq \mathrm{X}$ is defined as follows:

$$
\mathrm{A}=\left\{\left(\mathrm{x}, \mathrm{y}, \xi_{\mathrm{A}}(\mathrm{x}, \mathrm{u}): \mathrm{x} \in \mathrm{X}, \mathrm{u} \in[0,1]\right\},\right.
$$

where $\xi_{\mathrm{A}}(\mathrm{x}, \mathrm{u})=1, \mathrm{x} \in \mathrm{X}, \mathrm{u} \in[0,1]$.

Type-2 Fuzzy Sets have been widely and fruitfully used in a clustering context to manage different kinds of uncertainty affecting the clustering process. In particular, Type-2 Fuzzy Sets have been used in clustering for managing uncertainty related to the value of suitable parameters of the clustering algorithms. Ozkan and Turksen (2004) considered uncertainties of various parameters from imperfect information of patterns when applying fuzzy c-means. In particular, they focused on the uncertainty regarding the fuzziness parameter $m$ according to entropies after removing uncertainties from the other parameters (e.g. number of clusters, cluster centers, and so on). Rhee and Hwang published several papers on uncertain fuzzy clustering based on IT2FS; i.e. in these papers they discussed the extension of several T1FS-based clustering methods (FcM) into IT2FS, namely the IT2 fuzzy perceptron (Rhee and Hwang, 2002 [130]), the IT2 fuzzy k-nearest neighbor algorithm [253], the IT2 fuzzy c-spherical shell algorithm [131], and IT2 FcM algorithm ([251,252,254]). In all papers published in period 2002-2004, they studied on how to define and manage uncertainty for distance measures when fuzzy membership functions are designed in clustering. In 2007, Hwang and Rhee [359] proposed the so-called Interval Type-2 Fuzzy FCM (IT2FCM) clustering method. In particular, they focused their attention on the representation and management of uncertainty which is present in the fuzzy memberships of the pattern associated to the varying of fuzzifier parameter $m$ that controls the amount of fuzziness of the final fuzzy partition obtained by means of FCM. For designing and managing the uncertainty for $m$, they extended a pattern set to interval Type-2 fuzzy sets utilizing two fuzzifier $m_{1}$ and $m_{2}$ which creates a footprint of uncertainty (FOU) for the fuzzifier parameter $m$. Then, they incorporated this interval Type-2 fuzzy set into FcM to observe the effect of managing uncertainty from the two fuzzifiers. They computed the upper and lower membership $\left[\underline{u}_{i k}, \bar{u}_{i k}\right]$ and successively the estimated centers by using the centroid type-reduction represented by the interval $\left[\mathrm{v}_{\mathrm{L}}, \mathrm{V}_{\mathrm{R}}\right]$. The KarnikMendel (KM) iterative algorithm was proposed to estimate the left $\mathrm{v}_{\mathrm{L}}$ and right $\mathrm{v}_{\mathrm{R}}$ for an interval cluster center when performing type-reduction during center-updating (see, Hwang and Rhee, [359]).

Min et al. [198] suggested an IT2F Possibilistic c-means clustering (IT2FPcM) incorporating IT2Fss into the Possibilistic c-means clustering (PcM). They defined upper and lower memberships utilizing two different fuzzifiers $m_{1}$ and $m_{2}$ (see [198]).

Ji et al. [134] incorporated the IT2Fs into the hybrid fuzzy clustering scheme (fuzzy-possibilistic scheme), and thus proposed the interval Type-2 fuzzy possibilistic c-means (IT2FPcM) clustering algorithm. They used both fuzzy memberships and possibilistic typicalities to model the uncertainty implied in the data sets, and develop solutions to overcome the difficulties caused by Type- 2 fuzzy sets, such as the construction of footprint of uncertainty, type-reduction and defuzzification. 
Table 5

Contributes on Type-2 fuzzy clustering.

\begin{tabular}{ll}
\hline Rhee and Hwang [252] & $\begin{array}{l}\text { Sanchez et al. [264] } \\
\text { Golsefid and Zarandi [113] } \\
\text { Golsefid et al. [114] } \\
\text { Rguyen and Nahavandi [215] }\end{array}$ \\
Rhee and Hwang [253] & $\begin{array}{l}\text { Pham et al. [243] } \\
\text { Hwang and Rhee [131] }\end{array}$ \\
Rhee [251] & Rubio et al. [258] \\
Hwang and Rhee [359] [259] & Wu and Liu [309] \\
Min et al. [198] & Yao et al. [324] \\
Ji et al. [134] & Comas et al. [60] \\
Linda and Manic [166] & Rubio and Castillo [257] \\
Rubio and Castillo [256] & \\
\hline
\end{tabular}

In particular, they defined the lower and upper interval fuzzy membership and possibilistic typicality using, respectively, fuzzifiers $\left(m_{1}, m_{2}\right)$ and $\left(p_{1}, p_{2}\right)$. Then, they computed the interval of a primary fuzzy membership as $\left[\underline{u}_{i k}, \bar{u}_{i k}\right]$ and the interval of a primary possibilistic typicality as $\left[\underline{t}_{i k}, \bar{t}_{i k}\right]$ (see, [134]).

Note that all the previous clustering methods are based on a particular case of T2FSs, i.e. the IT2FSs. Although the original T2FSs may be useful in modeling uncertainty, the operations of T2FSs involve numerous embedded T2FSs, and thus require undesirably large amount of computations to consider all possible combinations of secondary membership values. Therefore, IT2FSs were proposed to reduce the computational complexity [195]. For this reason, i.e. because of the computational intensity of the processing of T2FSs, only their constrained version, i.e., the interval T2 (IT2) FSs, were typically used in cluster analysis. Fortunately, the recently introduced concepts of $\alpha$-planes and zSlices allow for efficient representation and computation with T2FSs. Following this development, Linda and Manic [166] proposed a new approach for uncertain fuzzy clustering using the T2FSs in the fuzzy c-means clustering, called Type-2 Fuzzy C-means clustering (T2FCM) or General Type2 Fuzzy C-means clustering (GT2FCM). The proposed method builds on top of the previously published IT2 FCM algorithm, which is extended via the $\alpha$-planes representation theorem [166].

In particular, Linda and Manic [166] proposed a novel method for managing the uncertainty associated with the selection of the fuzzifier parameter $m$ for the FCM algorithm, a selection that has a direct impact on the location and quality of the cluster partition.

The original T1FCM algorithm requires the specification of a precise fuzzifier value $m$. The IT2FCM algorithm accepts an interval-valued fuzzifier $\left[m_{L}, m_{R}\right]$, which resembles a uniform uncertainty about the appropriate value of fuzzifier $m$. The T2FCM algorithm proposed by Linda and Manic [166] accepts a linguistic description of the fuzzifier value expressed as a T1 fuzzy set (e.g., "small" or "high" ). The resulting cluster membership functions are implemented as T2 FSs represented using the $\alpha$-planes theorem. A novel hard-partitioning rule is proposed for the final input-cluster assignment. In addition, the quasi-T2 (QT2)FCM algorithm is also introduced as a simplified version of the GT2FCM method. See, for more detail, Linda and Manic [166].

Other recent interesting Type-2 fuzzy clustering methods have been suggested by Dang et al. [83], Rubio and Castillo [256], Sanchez et al. [264], Golsefid and Zarandi [113], Golsefid et al. [114], Nguyen and Nahavandi [215], Pham et al. [243], Rubio et al. [258,259], Wu and Liu [309], Yao et al. [324], Comas et al. [60], Rubio and Castillo [257].

A chronological list of the various contributes on Type-2 fuzzy clustering is shown in Table 5.

Summing up, in line with the Information-Uncertainty perspective, clustering based on IT2Fs and T2FS manage the uncertainty associated with fuzzifier parameter $m$ using an interval-valued fuzzifier $\left[m_{L}, m_{R}\right]$, which resembles a uniform uncertainty about the appropriate value of fuzzifier $m$ (for the IT2Fs case) and linguistic terms such as "small" or "high," which are modeled as T1FSs (for the T2FS case).

\subsection{Neutrosophic clustering}

Neutrosophic Sets (NSs) theory was introduced by Smarandache [274] as a generalization of Fuzzy Sets (FSs) and Intuitionistic Fuzzy Sets (IFSs) based on neutrosophy, which is a branch of philosophy. Neutrosophic sets (NSs) were proposed as a new branch of philosophy dealing with the origin, nature and scope of neutralities, and their interactions with different ideational spectra [275]. As illustrated in Guo and Şengür [360], element $<\mathrm{E}>$ in neutrosophic set is considered in relation to its opposite, $<$ Anti-E $>$ and its neutrality $<$ Neut-E $>$, which is neither $<\mathrm{E}>$ nor $<$ Anti-E $>$, and three memberships are employed to measure the degree of truth, indeterminacy and falsity of $<\mathrm{E}>$. Thus, NSs can not only evaluate the degree to true or false but also the degree to indeterminacy. Based on this character, neutrosophic theory provides a powerful tool to deal with indeterminacy, and has found practical applications in a variety of different fields (Guo and Şengür, [360]).

Formally, a Single-Valued Neutrosophic Set (SVNS) can be defined as follows. Let X be an initial universe. A Single-Valued Neutrosophic Set (SVNS) A $\subseteq \mathrm{X}$ is defined as follows:

$$
A=\left\{\left(x, t_{A}(x), i_{A}(x), f_{A}(x): x \in X\right\}\right.
$$


Table 6

Contributes on neutrosophic set-based clustering.

\begin{tabular}{ll}
\hline Shan et al. [269] & Guo and Şengür [119] \\
Yu et al. [330] & Guo and Şengür [120] \\
Anter et al. [13] & Alsmadi [9] \\
M.F. [13] & Guo et al. [121] \\
Guo and Şengür [118] & Huang [127] \\
Ye [327] & Karaaslan [141] \\
Ye [328] & Koundal et al. [149] \\
Akhtar and Ahmad [4] & Ye [329] \\
\hline
\end{tabular}

where $t_{A}(x)$ represents the truth-membership degree of $x$ to $A, i_{A}(x)$ indicates the indeterminacy-membership degree of $x$ to $A$ and $f_{A}(x)$ denotes the falsity-membership degree of $x$ to $A$ with the constraints:

$$
\mathrm{t}_{\mathrm{A}}(\mathrm{x}) \in[0,1], \mathrm{i}_{\mathrm{A}}(\mathrm{x}) \in[0,1], \mathrm{f}_{\mathrm{A}}(\mathrm{x}) \in[0,1] \text { and } 0 \leq \mathrm{t}_{\mathrm{A}}(\mathrm{x})+\mathrm{i}_{\mathrm{A}}(\mathrm{x})+\mathrm{f}_{\mathrm{A}}(\mathrm{x}) \leq 3, \forall \mathrm{x} \in \mathrm{X} \text {. }
$$

Although the theory of the Neutrosophic Sets was introduced eighteen years ago, it began to be used only recently in Statistics Reasoning and in cluster analysis, in particular.

More specifically, the theory has been recently applied to clustering for taking into account suitably the uncertainty affecting the clustering process. Among the most relevant contributes we have the neutrosophic c-means clustering method proposed by Guo and Şengür [119]. In particular, Guo and Şengür [119] suggested a neutrosophic set-clustering method, in which the degrees belonging to the determinant and indeterminate clusters are computed at the same time for each of the data points. They considered in the clustering procedure a membership degree to determinant clusters and other two memberships to determine two kinds of indeterminate clusters: an ambiguity cluster and an outlier cluster for each data point, respectively. "Ambiguity cluster allows us to consider about the data points that are laying near the clusters boundaries and outlier cluster allows us to reject individual data points when they are very far from the centers of each cluster. Both ambiguity and outlier clusters are introduced in the clustering iterations and not in the decision processing. The membership degrees to the ambiguity and outlier class of a data point are explicit, and these values are learned in the iterative clustering problem. So, the membership functions are more immune to noise and they correspond more closely to the notion of compatibility" [119]. By considering an extension of the classical Dempster-Shafer (DS) theory (see Section 4.8) -the so-called Dezert-Smarandache theory (DSmT) of plausible and paradoxical reasoning- Guo and Şengür [120] suggested a new clustering method called neutrosophic evidential c-means (NECM), based on NS and DSmT. In the NECM, the authors reformulate the objective function adopting a neutrosophic set to choose a suitable method for determining the mass function in the DSmT evidence theory. NS computes the truth degree (T), falsity degree (F), and indeterminacy degree (I) for each of the data points. While $\mathrm{T}$ is used as the membership degree in clustering algorithms, I and $\mathrm{F}$ are considered to define an ambiguity cluster and an outlier cluster, respectively, for each data point. The ambiguity cluster allows us to consider the data points that are lying near the clusters boundaries, and outlier cluster allows us to reject individual data points when they are very far from the centers of each cluster. The final belonging decision for each data point is transformed into an information fusion problem solved by DSmT. A new mass function was defined using membership degree, ambiguity degree, and outlier degree. The DSmT combination rule and decision are applied to obtain the final clustering result [120]. Akhtar and Ahmad [4] suggested a modified fuzzy c-means clustering using meutrosophic logic. Karaaslan [141] defined a new structure called Single-Valued Neutrosophic Refined Soft Set (SVNRSS) e two correlation coefficients for SVNRSS. Thus, the author suggested a clustering method based on these correlation coefficients.

The contributes on neutrosophic clustering suggested in the literature (most of the papers are very recent) are listed chronologically in Table 6.

Thus, in line with the Information-Uncertainty perspective, by means of Neutrosophic Sets-based clustering, the uncertainty associated with clustering process is managed by means of three types of membership degrees, i.e. truth-membership degree, indeterminacy-membership degree and falsity-membership degree.

\subsection{Hesitant fuzzy clustering}

Torra and Narukawa [290] and Torra [289] suggested a new extension of fuzzy sets so-called Hesitant Fuzzy Sets (HFSs), to deal with the difficulty that often arises when the membership degree of an element must be established, a difficulty not due to the error margin (as in intuitionistic fuzzy sets, IFS) or to possibility distribution (as in Type-2 fuzzy sets, T2FS), but rather to the fact that there are some possible values that create a hesitation over which to choose [255]. Then, Hesitant Fuzzy Sets are an extension of Fuzzy Sets in which the membership degree of a given object is defined as e asset of possible values. In particular, a Hesitant Fuzzy Set can be defined as follows.

Let $\mathrm{X}$ be an initial universe. A Hesitant Fuzzy Set(HFS) $A \subseteq X$ is defined as follows:

$$
A=\left\{\left(x, h_{A}(x): x \in X\right\}\right.
$$

where $h_{A}(x)$ is a set of different values in [0,1], representing possible membership degrees of $x$ to $A$.

Hesitant Fuzzy Sets theory can be usefully utilized for managing the uncertainty affecting different components of information in the clustering process. In fact, in group decision situations, the information provided by different decision makers 
(experts) can vary significantly. The standard fuzzy clustering schemes are unable to incorporate the differences in the opinions of different decision makers, that is, they are unsuitable for clustering in hesitant fuzzy environments. HFSs can be used to solve the issue, because they avoid performing data aggregation and can directly reflect differences of opinion among decision makers [315]. In this connection, many distance and similarity measures useful for clustering based on for HFSs are listed in Xu [315]. In particular, Xu and Xia [318] defined different distance measures for hesitant fuzzy sets. Chen et al. [55] derived a number of correlation coefficient formulas for HFSs and applied them to clustering analysis under hesitant fuzzy environments. Zhang and $\mathrm{Xu}$ [312] extended the agglomerative hierarchical clustering algorithm for classifying hesitant fuzzy information. Chen et al. [54] derived a number of correlation coefficient formulas for HFSs and applied them to clustering analysis under hesitant fuzzy environments. Chen et al. [54] investigated the clustering technique for HFSs based on the k-means clustering algorithm which takes the results of hierarchical clustering as the initial input. Zhang and Xu [342] suggested a hesitant fuzzy minimal spanning tree (HFMST) clustering algorithm under hesitant fuzzy environment.

Zhang and Xu [343] proposed a novel concept of hesitancy index of hesitant fuzzy set to measure the hesitancy degree among the possible values in each hesitant fuzzy element of the hesitant fuzzy set. By taking into account their hesitancy indices, they suggested new methods for measuring the distances between hesitant fuzzy sets and discuss their properties. Successively, according to the relationship between the distance measure and the similarity measure, they proposed two novel similarity measures for hesitant fuzzy sets and then define a novel hesitant fuzzy clustering algorithm on the basis of the novel similarity measures for classifying objects with hesitant fuzzy sets. Zhang and Xu [344] proposed a novel hesitant fuzzy agglomerative hierarchical clustering algorithm for HFSs. The algorithm considers each of the given HFSs as a unique cluster in the first stage, and then compares each pair of the HFSs by utilizing the weighted Hamming distance or the weighted Euclidean distance. Aliahmadipour et al. [6] defined a method to construct H-fuzzy partitions from a set of fuzzy clusters obtained from several executions of fuzzy clustering algorithms with various initializations of their parameters. Other hesitant fuzzy clustering methods have been suggested recently by Aliahmadipour and Eslami [5] and Torra et al. [291].

In line with the Information-Uncertainty perspective, the Hesitant Fuzzy Sets-based clustering methods take into account uncertainty by means of a set of different values, representing possible membership degree measures.

\subsection{Interval-based fuzzy clustering}

In the literature, there are several clustering methods based on a standard (non fuzzy) approach for managing imprecise data represented in the form of interval-valued data. See for instance de Souza and de Carvalho [88]; Billard and Diday [36].

Also as part of the fuzzy approach, many clustering methods for classifying imprecise data modeled as interval data have been suggested in the literature. See, e.g., de Carvalho and Tenorio (2004); D'Urso and Giordani (2006); D'Urso et al. [78,79]; D'Urso and Leski [82]. In all these methods the uncertainty is measured by point values of the membership degrees.

Two interesting clustering methods -based on the interval-valued sets theory- have been proposed by Silva et al. [273]. In particular, they proposed an interval distance measure and -combining the idea of interval distance and interval arithmetic in the clustering process- two clustering methods in which the membership degrees are interval-based values; thus, in these methods the uncertainty is measured by means of intervals of membership degrees.

\subsection{Picture fuzzy clustering}

Recently, Cuong and Kreinovich [73] introduced the concept of Picture Fuzzy Sets (PFSs) as extensions of the Fuzzy Sets (FSs) and the Intuitionistic Fuzzy Sets (IFSs). Formally, PFS can be defined as follows.

Let $X$ be an initial universe. A Picture Fuzzy Set (PFS) $A \subseteq X$ is defined as follows:

$$
\mathrm{A}=\left\{\left(\mathrm{x}, \mu_{\mathrm{A}}(\mathrm{x}), \eta_{\mathrm{A}}(\mathrm{x}), v_{\mathrm{A}}(\mathrm{x}): \mathrm{x} \in \mathrm{X}\right\},\right.
$$

where $\mu_{A}(x)$ represents the degree of positive membership of $x$ to $A, \eta_{A}(x)$ indicates the degree of neutral membership of $\mathrm{x}$ to $\mathrm{A}$ and $v_{\mathrm{A}}(\mathrm{x})$ denotes degree of negative membership of $\mathrm{x}$ to $\mathrm{A}$ with the constraints:

$$
\mu_{\mathrm{A}}(\mathrm{x}) \in[0,1], \eta_{\mathrm{A}}(\mathrm{x}) \in[0,1], v_{\mathrm{A}}(\mathrm{x}) \in[0,1] \text { and } 0 \leq \mu_{\mathrm{A}}(\mathrm{x})+\eta_{\mathrm{A}}(\mathrm{x})+v_{\mathrm{A}}(\mathrm{x}) \leq 1, \forall \mathrm{x} \in \mathrm{X}
$$

and then

$$
\mathrm{r}_{\mathrm{A}}(\mathrm{x})=1-\left(\mu_{\mathrm{A}}(\mathrm{x})+\eta_{\mathrm{A}}(\mathrm{x})+v_{\mathrm{A}}(\mathrm{x})\right)
$$

represents the degree of refusal membership of $x$ to $A$.

For more details on PFSs see Cuong and Kreinovich [73] and Cuong [72].

More recently, a number of clustering methods based on PFSs have been proposed. Son [277] proposed a distributed picture fuzzy clustering method on PFSs. Thong and So [284] proposed a hybrid method systems for medical diagnosis intermediate between picture fuzzy clustering and intuitionistic fuzzy recommender. Successively, the same authors have published other contributions to the picture fuzzy clustering method [278,279,281,285,286,286,287].

From an information-uncertainty viewpoint, the uncertainty in the picture fuzzy clustering is managed by means of the different types of membership degrees, i.e. degree of positive membership, degree of neutral membership and degree of negative membership. 
Table 7

Seminal papers on the different uncertain clustering approaches and connected papers on the uncertainty theories (ordered with respect to the first paper of each clustering approach).

\begin{tabular}{|c|c|c|c|}
\hline Year & Uncertain clustering approaches & Year & Uncertainty theories \\
\hline & Fuzzy clustering & & Fuzzy Sets (FSs) theory \\
\hline 1966 & Bellman, Kalaba, Zadeh [33] & 1965 & Zadeh [332] \\
\hline 1969 & Ruspini [260] & & \\
\hline 1970 & Ruspini [261] & & \\
\hline 1973 & Ruspini [262] & & \\
\hline 1974 & Bezdek [35] & & \\
\hline 1974 & Dunn $[356]$ & & \\
\hline \multirow[t]{2}{*}{1981} & Bezdek [355] & & \\
\hline & Possibilistic clustering & & Possibilistic (Poss) theory \\
\hline 1993 & Krishnapuram and Keller [152] & 1978 & Zadeh [333] \\
\hline \multirow[t]{2}{*}{1996} & Krishnapuram and Keller [152] & & \\
\hline & Shadowed clustering & & Shadowed Sets (SSs) theory \\
\hline 1998 & Pedrycz [225] & 1998 & Pedrycz [225] \\
\hline 2010 & Mitra et al. [204] (shadowed clustering integrating fuzzy and rough clustering) & & \\
\hline \multirow[t]{2}{*}{2011} & Zhou et al. [135] (shadowed set-based rough-fuzzy clustering) & & \\
\hline & Rough set-based clustering & & Rough Sets (RSs) theory \\
\hline 2004 & Lingras and West [171] (rough clustering) & 1982 & Pawlak [221] \\
\hline 2006 & Peters [236] (refined rough clustering) & & \\
\hline 2006 & Mitra, Banka, Pedrycz [206] (rough fuzzy clustering) & & \\
\hline 2007 & Maji and Pal [183] (rough fuzzy clustering) & & \\
\hline \multirow[t]{2}{*}{2007} & Maji and Pal [184] (rough fuzzy possibilistic clustering) & & \\
\hline & Intuitionistic fuzzy clustering & & Intuitionistic Fuzzy Sets (IFSs) theory \\
\hline 2004 & Hung et al. [130] & 1986 & Atanassov [25] \\
\hline 2008 & Pelekis et al. [234] & & \\
\hline 2008 & Torra et al. [292] & & \\
\hline \multirow[t]{2}{*}{2008} & $\mathrm{Xu}$ et al. [316] & & \\
\hline & Evidential clustering or credal clustering or Belief clustering & & Credal Sets (CSs) theory, Dempster- \\
\hline 2004 & Denœux, Masson [98] & 1967 & Shafer (DS) theory \\
\hline \multirow[t]{3}{*}{2008} & Denœux, Masson [190] & 1976 & Dempster, [90], Shafer [268] \\
\hline & & 1997 & Cozman $[68]$ \\
\hline & Credibilistic clustering & & Credibilistic (Cred) theory \\
\hline 2007 & Zhou et al. [350] & 2004 & Liu [174] \\
\hline 2014 & Niakan et al. [216] & & \\
\hline \multirow[t]{2}{*}{2015} & Zhou et al. [348] & & \\
\hline & Type-2 fuzzy clustering & & Type-2 Fuzzy Sets (T2FSs) theory \\
\hline 2007 & Hwang and Rhee [359] (interval Type-2 fuzzy clustering) & 1975 & Zadeh [353] \\
\hline 2009 & Min et al. [198] (interval Type-2 possibilistic clustering) & & \\
\hline 2012 & Linda and Manic [166] (Type-2 fuzzy clustering or general Type-2 fuzzy clustering) & & \\
\hline \multirow{2}{*}{2014} & Ji et al. [134] (interval Type-2 fuzzy possibilistic clustering) & & \\
\hline & Neutrosophic clustering & & Neutrosophic Sets (NSs) theory \\
\hline 2012 & Shan et al. [269] & 1998 & Smarandache [274] \\
\hline \multirow[t]{2}{*}{2015} & Guo and Şengür [119] & & \\
\hline & Hesitant fuzzy clustering & & Hesitant Fuzzy Sets (HFSs) theory \\
\hline \multirow[t]{2}{*}{2013} & Chen et al. [55] & 2009 & Torra and Narukawa [290] \\
\hline & Interval-based fuzzy clustering & & Interval Sets (ISs) theory \\
\hline 2015 & Silva et al. [273] & 1966 & Moore [211] \\
\hline \multirow[t]{2}{*}{2015} & Picture fuzzy clustering & 2013 & Picture Fuzzy Sets (PFSs) theory \\
\hline & Son [277] & & Cuong and Kreinovich [73] \\
\hline
\end{tabular}

\section{A final summary overview}

As we have seen in the previous sections, after the first methods of fuzzy clustering several methods based on different approaches to manage uncertainty have been proposed.

To offer an overview of the time interval in which these various uncertain clustering methods have been proposed, we show in Fig. 10 the timeline of the first more relevant papers on the various uncertainty clustering approaches and the first papers dealing with related uncertainty theories.

In Table 7, we have ordered chronologically the seminal papers on the various uncertain clustering approaches and the related papers on uncertainty theories (the chronological order is with respect to the first paper on the different clustering approaches).

In Fig. 10 and Table 7, we note that in some cases the time gap between the year of the first paper on the uncertainty theory and the year of the first paper on the connected uncertain clustering approach is short (e.g. the case of Fuzzy Sets with the fuzzy clustering) and sometimes very long (e.g., that is the case of Rough Sets, Intuitionistic Fuzzy Sets, Belief theory, Type-2 Fuzzy Sets and Neutrosophic Sets with the respective uncertainty theories). 


\section{Clustering approaches}

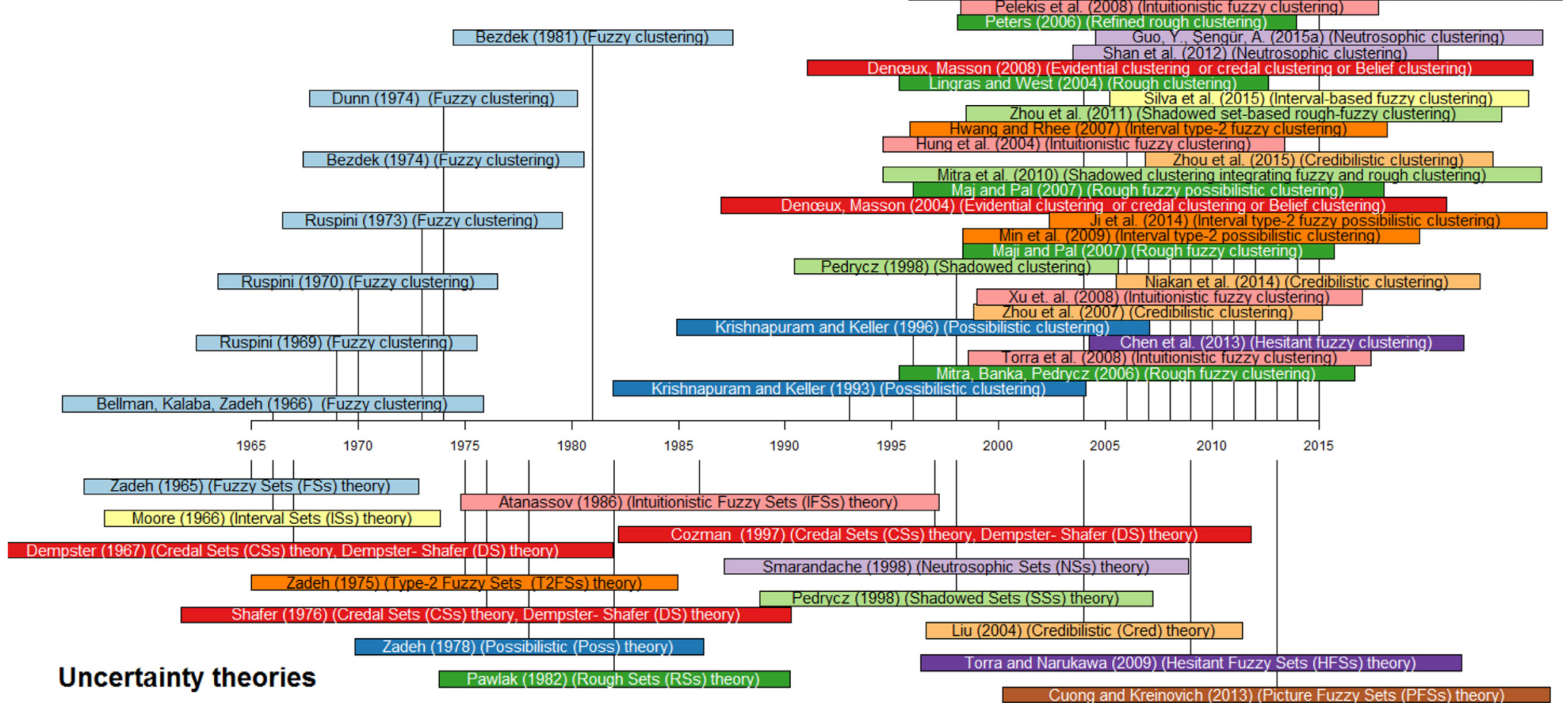

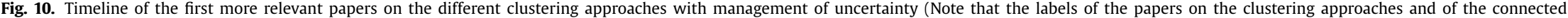
papers on the uncertainty theories have the same color). We remark that we do not consider clustering approaches based on the standard probability theory. 
Table 8

Clustering approaches, theoretical formalisms and uncertainty measures.

\begin{tabular}{|c|c|c|}
\hline Uncertain theoretical formalisms & Uncertain clustering approaches & Uncertain measures \\
\hline Fuzzy Sets (FSs) & Fuzzy clustering & point membership degrees \\
\hline \multirow[t]{2}{*}{ Possibilistic theory } & Possibilistic clustering & point possibility degrees (or compatibility degrees) \\
\hline & Hybrid clustering (Fuzzy-Possibilistic clustering) & point membership degrees and typicality degrees \\
\hline Interval Sets (ISs) and Fuzzy Sets (FSs) & Interval-based fuzzy clustering & intervals of membership degrees \\
\hline $\begin{array}{l}\text { Dempster- Shafer (DS) theory and } \\
\text { Credal Sets (CSs) }\end{array}$ & $\begin{array}{l}\text { Evidential clustering or credal clustering or Belief } \\
\text { clustering }\end{array}$ & Dempster-Shafer mass functions or degree of belief \\
\hline \multirow[t]{4}{*}{$\begin{array}{l}\text { Type-2 Fuzzy Sets (IT2FSs) and Interval } \\
\text { Type-2 Fuzzy Sets (T2FSs) }\end{array}$} & Interval Type-2 fuzzy clustering & $\begin{array}{l}\text { upper and lower fuzzifier parameters, interval } \\
\text { fuzzifier parameters, upper and lower } \\
\text { membership degrees, interval membership } \\
\text { degrees, footprint of uncertainty }\end{array}$ \\
\hline & Interval Type-2 possibilistic clustering & $\begin{array}{l}\text { upper and lower fuzzifier parameters, interval } \\
\text { fuzzifier parameters, upper and lower typicality } \\
\text { degrees, interval typicality degrees, footprint of } \\
\text { uncertainty }\end{array}$ \\
\hline & Interval Type-2 fuzzy-possibilistic clustering & $\begin{array}{l}\text { upper and lower fuzzifier parameters for } \\
\text { membership and typicality degrees, interval } \\
\text { fuzzifier parameters, upper and lower } \\
\text { membership degrees, upper and lower typicality } \\
\text { degrees, interval memebrship degrees interval } \\
\text { typicality degrees, footprint of uncertainty }\end{array}$ \\
\hline & Type-2 fuzzy clustering & $\begin{array}{l}\text { linguistic fuzzifier parameter, } \alpha \text {-plane of } \\
\text { membership degrees }\end{array}$ \\
\hline Intuitionistic Fuzzy Sets (IFSs) & Intuitionistic fuzzy clustering & $\begin{array}{l}\text { belongingness degrees (membership degrees) and } \\
\text { non-belongingness degrees (non-membership } \\
\text { degrees) }\end{array}$ \\
\hline \multirow{2}{*}{$\begin{array}{l}\text { Rough Sets (RSs) and Rough Fuzzy Sets } \\
\text { (RFSs) }\end{array}$} & Rough clustering & crisp lower and upper approximations \\
\hline & $\begin{array}{l}\text { Rough Fuzzy clustering and Rough Fuzzy } \\
\text { Possibilistic clustering }\end{array}$ & $\begin{array}{l}\text { fuzzy and/or crisp lower and upper } \\
\text { approximations, fuzzy boundary, } \\
\text { fuzzy-possibilistic boundary }\end{array}$ \\
\hline Neutrosophic Sets (NSs) & Neutrosophic clustering & $\begin{array}{l}\text { truth-membership degrees, } \\
\text { indeterminacy-membership degrees, } \\
\text { falsity-membership degrees }\end{array}$ \\
\hline Shadowed Sets (SSs) & Shadowed clustering & exclusion, shadowed, and core zones \\
\hline Credibilistic theory & Credibilistic clustering & credibilistic measures \\
\hline Hesitant Fuzzy Sets (HFSs) & Hesitant fuzzy clustering & $\begin{array}{l}\text { membership degrees defined as e asset of possible } \\
\text { values }\end{array}$ \\
\hline Picture Fuzzy Sets (PFSs) & Picture fuzzy clustering & $\begin{array}{l}\text { positive membership degrees, neutral membership } \\
\text { degrees, negative membership degrees }\end{array}$ \\
\hline
\end{tabular}

All this highlights the different timing of the impacts of the various theories on uncertainty from the respective of methodological approaches adopted for clustering objects in uncertainty conditions; thus, the metabolic process of the theoretical results used in the respective clustering methodologies has been different.

In Table 8, we summarize for each uncertain clustering approach, the connected theoretical formalism from which they are inspired and the measures adopted for managing the uncertainty in the clustering process.

\section{Conclusions}

In Statistical reasoning, uncertainty affects the empirical and theoretical information of the knowledge process.

For this reason, in the literature, various theoretical platforms have been proposed for formalizing, measuring and modeling the uncertainty. Focusing on the Fuzzy Sets theory [332] and its extensions and derivatives, in this paper the specific area of Cluster Analysis has been explored.

In the last 50 years, after the first paper on fuzzy clustering [33], many clustering methods have been proposed. In fact, following different theoretical approaches for managing the uncertainty affecting the classification process, many extensions and derivatives of fuzzy clustering have been suggested.

In this connection, using the various uncertainty theories, in each clustering approach, suitable uncertainty measures have been defined for managing uncertainty affecting the empirical and theoretical informational ingredients of clustering methodology.

In this paper, we have presented an organic and systematic literature review of different uncertain clustering approaches, i.e. Fuzzy clustering, Possibilistic clustering, Shadowed clustering, Rough sets-based clustering, Intuitionistic fuzzy clustering, Evidential clustering, Credibilistic clustering, Type-2 fuzzy clustering, Neutrosophic clustering, Hesitant fuzzy clustering, Interval-based fuzzy clustering, and Picture fuzzy clustering.

In the future, it could be interesting to design - in a clustering framework and also in a more general theoreticalmethodological context- a theoretical platform and to organize an integrated system in which the several theoretical ap- 
proaches for managing the different types of uncertainty are synergically considered. In this connection, the ultimate goals are: to suitably model uncertainty through innovative and more efficient uncertainty measures, to reduce uncertainty and informational redundancy, to improve the informational gain and to optimize the power of the different theoretical formalisms.

These will be ones of the most exciting challenges in coming years.

In this respect, Granular Computing could have a relevant role in establishing a stimulating synergy between individual theoretical approaches [224].

However, although the Statistical Reasoning System may be an effective and successful approach, a halo of uncertainty will always permeate information and therefore knowledge. The only certainty is that there is no certainty.

\section{Acknowledgments}

I wish to thank the editor Prof. Witold Pedrycz and the referees for their useful comments and suggestions which helped to improve the quality and presentation of this paper. I dedicate this paper to the memory of my father.

\section{References}

[1] A. Abadpour, Rederivation of the fuzzy-possibilistic clustering objective function through Bayesian inference, Fuzzy Sets Syst. 305 (2016) 29-53.

[2] A. Abadpour, Incorporating spatial context into fuzzy-possibilistic clustering using Bayesian inference, J. Intell. Fuzzy Syst. 30 (2016) 895-919.

[3] S. Agrawal, B.K. Tripathy, Decision theoretic rough intuitionistic fuzzy C-means algorithm, smart innovation, systems and technologies, in: International Conference on Information and Communication Technology for Intelligent Systems, ICTIS 2015, 28-29 November 2015, Ahmedabad, India, Springer, 2016, pp. 71-82.

[4] N. Akhtar, M.V. Ahmad, A modified fuzzy c-means clustering using neutrosophic logic, in: Proceedings of - 2015 th International Conference on Communication Systems and Network Technologies, CSNT 2015, India, IEEE press, 2015, pp. 1124-1128.

[5] L. Aliahmadipour, E. Eslami, GHFHC: generalized hesitant fuzzy hierarchical clustering algorithm, Int. J. Intell. Syst. 31 (2016) 855-871.

[6] L. Aliahmadipour, V.Eslami Torra, M. Eftekhari, A definition for hesitant fuzzy partitions, Int. J. Comput. Intell. Syst. 3 (2016) 497-505.

[7] S. Alkhazaleh, A.R. Salleh, N. Hassan, Soft multisets theory, Appl. Math. Sci. 5 (2011) 3561-3573.

[8] Alkhazaleh, S., Salleh, A.R. (2012) Fuzzy soft multiset theory, abstract and applied analysis, article ID 350600, 20 pages.

[9] M.K. Alsmadi, A hybrid Fuzzy C-means and neutrosophic for jaw lesions segmentation, Ain Shams Eng. J. (2017) in press

[10] A. Amirkhani, M.R. Mosavi, K. Mohammadi, E.I. Papageorgiou, A novel hybrid method based on fuzzy cognitive maps and fuzzy clustering algorithms for grading celiac disease, Neural Comput. Appl. (2017) in press.

[11] A. Ammar, Z. Elouedi, P. Lingras, Meta-clustering of possibilistically segmented retail datasets, Fuzzy Sets Syst. 286 (2016) $173-196$.

[12] V.P. Ananthi, P. Balasubramaniam, T. Kalaiselvi, A new fuzzy clustering algorithm for the segmentation of brain tumor, Soft Comput. 20 (2016) 4859-4879.

[13] A.M. Anter, A.E. Hassanien, M.A.A. ElSoud, M.F. Tolba, Neutrosophic sets and fuzzy C-means clustering for improving CT liver image segmentation, Adv. Intell. Syst. Comput. 303 (2014) 193-203.

[14] V. Antoine, B. Quost, M.-H. Masson, T. Denœux, CECM: constrained evidential C-means algorithm, Comput. Stat. Data Anal. 56 (2012) 894-914.

[15] V. Antoine, B. Quost, M.-H. Masson, T. Denoeux, CEVCLUS: evidential clustering with instance-level constraints for relational data, Soft Comput. 18 (2014) 1321-1335

[16] J. Aparajeeta, P.K. Nanda, N. Das, Modified possibilistic fuzzy C-means algorithms for segmentation of magnetic resonance image, Appl. Soft Comput. 41 (2016) 104-119.

[17] S. Asharaf, S.K. Shevade, N.M. Murty, Rough support vector clustering, Pattern Recognit. 38 (2005) 1779-1783.

[18] S. Asharaf, S.K. Shevade, N.M. Murty, Rough set based incremental clustering of interval data, Recognit. Lett. 27 (2006) 515-519.

[19] S. Asharaf, M.N. Murty, An adaptive rough fuzzy single pass algorithm for clustering large data sets, Pattern Recognit. 36 (2003) 3015-3018.

[20] S. Askari, N. Montazerin, M.H.F. Zarandi, E. Hakimi, Generalized entropy based possibilistic fuzzy C-Means for clustering noisy data and its convergence proof, Neurocomputing 219 (2017) 186-202.

[21] K. Atanassov, Remark on the intuitionistic fuzzy sets-III, Fuzzy Sets Syst. 75 (1995) 401-402.

[22] K. Atanassov, Intuitionistic Fuzzy sets: Theory and Applications, Springer, Heidelberg, 1999.

[23] K. Atanassov, G. Gargov, Interval-valued intuitionistic fuzzy sets, Fuzzy Sets Syst. 31 (1989) 343-349.

[24] K. Atanassov, Answer to D. Dubois, S. Gottwald, P. Hajek, J. Kacprzyk and H. Prade's paper Terminological difficulties in fuzzy set theory-the case of Intuitionistic Fuzzy Sets, Fuzzy Sets Syst. 156 (2005) 496-499.

[25] K. Atanassov, Intuitionistic fuzzy sets, Fuzzy Sets Syst. 20 (1986) 87-96.

[26] S. Auephanwiriyakul, J.M. Keller, Analysis and efficient implementation of a linguistic fuzzy c-means, IEEE Trans. Fuzzy Syst. 10 (2002) 563-582.

[27] P. Balasubramaniam, V.P. Ananthi, Segmentation of nutrient deficiency in incomplete crop images using intuitionistic fuzzy C-means clustering algorithm, Nonlinear Dyn. 83 (2016) 849-866

[28] H. Bandemer, Mathematics of Uncertainty. Ideas, Methods, Application Problems, Springer-Verlag, Berlin, 2006.

[29] A. Bargiela, W. Pedrycz, Granular Computing: An Introduction, Kluwer Academic Publishers, Boston, 2003.

[30] M. Barni, R. Gualtieri, A new possibilistic clustering algorithm for line detection in real world imagery, Pattern Recognit. 32 (11) (1999) 1897-1909.

[31] M. Barni, V. Cappellini, A. Mecocci, Comments on “a possibilistic approach to clustering”, IEEE Trans. Fuzzy Syst. 4 (1996) 393-396.

[32] C. Bean, C. Kambhampati, Autonomous clustering using rough set theory, Int. J. Autom. Comput. 5 (2008) 90-102.

[33] R.E. Bellman, R. Kalaba, L. Zadeh, Abstraction and pattern classification, J. Math. Anal. Appl. 13 (1966) 1-7.

[34] J.P. Benzécri, L'analyse Des Données, Dunod, Paris, 1973.

[35] J.C. Bezdek, Numerical taxonomy with fuzzy sets, J. Math. Biol. 1 (1974) 57-71.

[36] L. Billard, E. Diday, Symbolic Data Analysis: Conceptual Statistics and Data Mining, Wiley, England, 2006.

[37] W.D. Blizard, Real-valued multisets and fuzzy sets, Fuzzy Sets Syst. 33 (1989) 77-97.

[38] J.G. Brown, A note on fuzzy sets, Inf. Control 18 (1971) 32-39.

[39] M. Burgin, Theory of named sets as a foundational basis for mathematics, in: Structures in Mathematical Theories, San Sebastian, 1990, pp. 417-420.

[40] H. Bustince, P. Burillp, Vague sets are intuitionistic fuzzy sets, Fuzzy Sets Syst. 79 (1996) 403-405.

[41] B.S. Butkiewicz, Robust fuzzy clustering with fuzzy data, in: Proceedings of Advances in Web Intelligence, Third International Atlantic Web Intelligence Conference, AWIC 2005, Lecture Notes in Computer Science, 352, Springer, 2005, pp. 76-82.

[42] C. Hennig, M. Meila, F. Murtagh, R. Rocci, Handbook of Cluster Analysis, Chapman \& Hall, 2015

[43] F. Cai, F.J. Verbeek, Rough fuzzy c-means and particle swarm optimization hybridized method for information clustering problem, J. Commun. 11 (2016) 1106-1113.

[44] R. Cai, Y.J. Lei, X.J. Zhao, Clustering method based on intuitionistic fuzzy equivalent dissimilarity matrix, J. Comput. Appl. 29 (2009) 123-126. 
[45] G. Cantor, "Ueber eine Eigenschaft des Inbegriffes aller reellen algebraischen Zahlen" ("On a Property of the Collection of All Real Algebraic Numbers"), J. Reine Angew. Math. 77 (1874) 258-262.

[46] O. Castillo, P. Melin, Type-2 Fuzzy Logic: Theory and Applications, Springer-Verlag, Heidelberg, 2008.

[47] T. Chaira, A novel intuitionistic fuzzy C means clustering algorithm and its application to medical images, Appl. Soft Comput. 11 (2011) $1711-1717$.

[48] T. Chaira, A. Panwar, An Atanassov's intuitionistic fuzzy kernel clustering for medical image segmentation, Int. J. Comput. Intell. Syst. 7 (2014) 360-370.

[49] S.-T. Chang, K.-P. Lu, M.-S. Yang, Stepwise possibilistic c-regressions, Inf. Sci. 334-335 (2016) 307-322.

[50] E.W. Chapin, Set-valued set theory, I, Notre Dame J. Formal Logic 15 (1974) 619-634.

[51] E.W. Chapin, Set-valued set theory, II, Notre Dame J. Formal Logic 16 (1975) 255-267.

[52] C.-B. Chen, L.-Y. Wang, Rough set-based clustering with refinement using Shannon's entropy theory, Comput. Math. Appl. 52 (2006) 1563-1576.

[53] J. Chen, C. Zhang, Efficient clustering method based on rough set and genetic algorith, Procedia Eng. 15 (2011) 1498-1503.

[54] N. Chen, Z.-S. Xu, M.-M. Xia, Hierarchical hesitant fuzzy K-means clustering algorithm, Appl. Math. 29 (2014) 1-17.

[55] N. Chen, Z. Xu, M. Xia, Correlation coefficients of hesitant fuzzy sets and their applications to clustering analysis, Appl. Math. Modell. 37 (2013) $2197-2211$.

[56] W. Chen, Z. Tang, X. Jiang, J. Gao, R. Sun, S. Hashlan, Agglomerative clustering using improved rough sets and its applications in cooperative object localization, Comput. Electr. Eng. 39 (2013) 1962-1969.

[57] X. Chen, Y. Liu, Correlation coefficients of intuitionistic hesitant fuzzy sets and their applications to clustering analysis, Int. J. Control Autom. 9 (2016) $403-418$.

[58] X. Cheng, R. Wu, Clustering path profiles on a website using rough k-means method, J. Comput. Inf. Syst. 8 (2012) 6009-6016.

[59] A. Colubi, G. Gonzales Rodriguez, P. D’Urso, M. Montenegro, Multi-sample test-based clustering for fuzzy random variables, Int. J. Approximate Reasoning 50 (2009) $721-731$.

[60] D.S. Comas, G.J. Meschino, A. Nowé, V.L. Ballarin, Discovering knowledge from data clustering using automatically-defined interval type-2 fuzzy predicates, Expert Syst. Appl. 68 (2017) 136-150

[61] R. Coppi, A theoretical framework for data mining: the "Informational Paradigm", Comput. Stat. Data Anal. 38 (2002) $501-515$.

[62] R. Coppi, Management of uncertainty in statistical reasoning: the case of regression analysis, Int. J. Approximate Reasoning 47 (2008) 284-305.

[63] R. Coppi, P. D’Urso, P. Giordani, Fuzzy and possibilistic clustering model for fuzzy data, Comput. Stat. Data Anal. (2012) $915-927$.

[64] R. Coppi, M.A. Gil, H.A.L. Kiers, The fuzzy approach to statistical analysis, Comput. Stat. Data Anal. 51 (2006) 1-14.

[65] C. Cornelis, M. De Cock, E.E. Kerre, Intuitionistic fuzzy rough sets: at the crossroads of imperfect knowledge, Expert Syst. Appl. 20 (2003) 260-270.

[66] C. Cornelis, C. Deschrijver, E.E. Kerre, Implication in intuitionistic and interval-valued fuzzy set theory: construction, classification, application, Int. J. Approximate Reasoning 35 (2004) 55-95.

[67] D.R. Cox, D. Hinkley, Theoretical Statistics, Chapman and Hall, London, 1974

[68] F.G. Cozman, Decision Making Based on Convex Sets of Probability Distributions: Quasi-Bayesian Networks and Outdoor Visual Position Estimation PhD thesis, The Robotics Institute, Carnegie Mellon University, 1997.

[69] F.G. Cozman, Credal networks, Artif. Intell. 120 (2000) 199-233.

[70] F.G. Cozman, Graphical models for imprecise probabilities, Int. J. Approximate Reasoning 39 (2005) 167-184.

[71] F. Crespo, G. Peters, R. Weber, Rough clustering approaches for dynamic environments, in: G. Peters, P. Lingras, D. Slezak, Y.Y. Yao (Eds.), Rough Sets: Selected Methods and Applications in Management and Engineering, Advanced Information and Knowledge Processing, Springer, London, 2012, pp. 39-50.

[72] C. Cuong, Picture fuzzy sets, J. Comput. Sci. Cybern. 30 (2014) 409-420.

[73] C. Cuong, V. Kreinovich, Picture fuzzy sets -a new concept for computational intelligence problems, in: Proceedings of the Third World Congress on Information and Communication Technologies WICT'2013, Hanio, Vietnam, 2013, pp. 1-6.

[74] P. D’Urso, L. De Giovanni, P. Spagnoletti, A fuzzy taxonomy for e-health projects, Int. J. Mach. Learn. Cybern. 4 (2013) $487-504$.

[75] P. D’Urso, in: J.V. de Oliveira, W. Pedrycz (Eds.), Fuzzy Clustering of Fuzzy Data, in “Advances in Fuzzy Clustering and Its Applications”, J. Wiley and Sons, 2007, pp. 155-192.

[76] P. D’Urso, L. De Giovanni, M. Disegna, R. Massari, Bagged clustering and its application to tourism market segmentation, Expert Syst. Appl. 40 (2013) 4944-4956.

[77] P. D’Urso, L. De Giovanni, Robust clustering of imprecise data, Chemom. Intell. Lab. Syst. 136 (2014) 58-80.

[78] P. D’Urso, L. De Giovanni, R. Massari, Trimmed fuzzy clustering for interval-valued data, Adv. Data Anal. Classification 9 (2015) 21-40.

[79] P. D'Urso, L. De Giovanni, R. Massari, C. Cappelli, Exponential distance-based fuzzy clustering for interval-valued data, Fuzzy Optim. Decis. Making 16 (2017) 51-70.

[80] P. D'Urso, P. Giordani, A weighted fuzzy c-means clustering model for fuzzy data, Comput. Stat. Data Anal. 50 (6) (2006) $1496-1523$.

[81] P. D’Urso, P. Giordani, A robust fuzzy k-means clustering model for interval valued data, Comput. Stat. 21 (2006) 251-269.

[82] P. D’Urso, J. Leski, Fuzzy C-ordered medoids clustering of interval-valued data, Pattern Recognit. 58 (2016) 9-67.

[83] T.H. Dang, L.T. Ngo, W. Pedrycz, Interval Type-2 fuzzy C-Means approach to collaborative clustering, in: IEEE International Conference on Fuzzy Systems (FUZZ-IEEE), 2015, Istanbul, 2015, pp. 1-7.

[84] M. De Càceres, F. Oliva, X. Font, On relational possibilistic clustering, Pattern Recognit. 39 (2006) 2010-2024.

[85] F.D.A. de Carvalho, C. Tenorio, Fuzzy k-means clustering algorithms for interval valued data based on adaptive quadratic distances, Fuzzy Sets Syst. 161 (2010) 2978-2999.

[86] F.D.A. de Carvalho, Fuzzy c-means clustering methods for symbolic interval data, Pattern Recognit. Lett. 28 (2007) $423-437$.

[87] J.V. de Oliveira, W. Pedrycz, Advances in Fuzzy Clustering and Its Applications, J. Wiley and Sons, 2007.

[88] R.M.C.R. de Souza, F.d.A. de Carvalho, Clustering of interval data based on city-block distances, Pattern Recognit. Lett. 25 (2004) $353-365$

[89] M. Demirci, Genuine Sets, Fuzzy Sets Syst. 105 (1999) 377-384.

[90] A.P. Dempster, Upper and lower probabilities induced by a multivalued mapping, Ann. Math. Stat. 38 (1967) $325-339$.

[91] T. Denœux, 2016. evclust: Evidential Clustering https://cran.r-project.org/web/packages/evclust/.

[92] T. Denœux, A k-nearest neighbor classification rule based on Dempster-Shafer theory, IEEE Trans. Syst. Man Cybern. Part B 34 (1995) 95-109.

[93] T. Denoeux, O. Kanjanatarakul, Evidential clustering: a review, lecture notes in computer science, in: 5th International Symposium on Integrated Uncertainty in Knowledge Modelling and Decision Making, IUKM 2016, 30 November 2016 - 2 December, Da Nang, Vietnam, 2016 , pp. 24-35.

[94] T. Denoeux, O. Kanjanatarakul, Beyond fuzzy, possibilistic and rough: an investigation of belief functions in clustering, in: Advances in Intelligent Systems and Computing, 8th International Conference on Soft Methods in Probability and Statistics, SMPS 2016, 12-14 September 2016, , Rome, Italy, Springer, 2017, pp. 157-164.

[95] T. Denœux, S. Sriboonchitta, O. Kanjanatarakul, Evidential clustering of large dissimilarity data, Knowl. Based Syst. 106 (2016) 179-195.

[96] T. Denœux, O. Kanjanatarakul, S. Sriboonchitta, EK-NNclus: a clustering procedure based on the evidential K-nearest neighbor rule, Knowl. Based Syst. 88 (2015) 57-69.

[97] T. Denœux, M.-H. Masson, EVCLUS: evidential clustering of proximity data, IEEE Trans. Syst. Man Cybern. Part B 34 (2004) $95-109$.

[98] T. Denœux, M.-H. Masson, Evidential reasoning in large partially ordered sets, Ann. Oper. Res. 195 (2012) $135-161$.

[99] G. Deschrijver, E.E. Kerre, On the relationship between some extensions of fuzzy set theory, Fuzzy Sets Syst 133 (2003) $227-235$.

[100] H.A. do Prado, P.M. Engel, H.C. Filho, Rough clustering: an alternative to find meaningful clusters by using the reducts from a dataset, in: Rough Sets and Current Trends in Computing: Third International Conference, RSCTC 2002, Lecture Notes in Computer Science, vol. 2475, Springer, Berlin, 2002, pp. 234-238. 
[101] Y.K. Dubey, M.M. Mushrif, K. Mitra, Segmentation of brain MR images using rough set based intuitionistic fuzzy clustering, Biocybern. Biomed. Eng. 36 (2016) 413-426.

[102] D. Dubois, H. Prade, Rough fuzzy sets and fuzzy rough sets, Int. J. Gen. Syst. 17 (1990) 191-209.

[103] D. Dubois, S. Gottwald, P. Hajek, J. Kacprzyk, H. Prade, Terminological difficulties in fuzzy set theory-The case of "Intuitionistic Fuzzy Sets", Fuzzy Sets Syst. 156 (2005) 485-491.

[104] D. Dubois, H. Prade, Bridging gaps between several forms of granular computing, Granular Comput. 1 (2016) 1115-1126.

[105] P. D’Urso, L. De Giovanni, R. Massari, Trimmed fuzzy clustering for interval-valued data, Adv. Data Anal. Classification 9 (2015) 21-40.

[106] F. Feng, Generalized rough fuzzy sets based on soft sets, Soft Comput. 14 (2010) 899-911.

[107] A. Gacek, W. Pedrycz, Clustering granular data and their characterization with information granules of higher type, IEEE Trans. Fuzzy Syst. 23 (4) (2015) 850-860.

[108] C. Gao, W. Pedrycz, D. Miao, Rough subspace-based clustering ensemble for categorical data, Soft Comput. Fusion Found. Methodol. Appl. 17 (2013) 1643-1658.

[109] W.L. Gau, D.J. Buehrer, Vague sets, IEEE Trans. Syst. Man Cybern. 23 (1993) 610-614

[110] Y. Gentilhomme, Les ensembles flous en linguistique, Cahiers Linguistique Theoretique Appliqee 5 (1968) $47-63$.

[111] I. Gitman, M. Levine, An algorithm for detecting unimodal fuzzy sets and its application as a clustering technique, IEEE Trans. Comp. C-19 (1970) $583-593$.

[112] J.A. Goguen, L-fuzzy Sets, J. Math. Anal. Appl. 18 (1967) 145-174.

[113] S.M.M. Golsefid, M.H.F. Zarandi, Dual-centers type-2 fuzzy clustering framework and its verificationand validation indices, Appl. Soft Comput. 47 (2016) 600-613.

[114] S.M.M. Golsefid, M.H.F. Zarandi, I.B. Turksen, Multi-central general type-2 fuzzy clustering approach for pattern recognitions, Inf. Sci. 328 (2016) $172-188$.

[115] I. Grattan-Guinness, Fuzzy membership mapped onto interval and many-valued quantities, Z. Math. Logik. Grundladen Math. 22 (1975) 149-160.

[116] P. Grzegorzewski, E. Mrowka, Some notes on (Atanassov's) intuitionistic fuzzy sets, Fuzzy Sets Syst. 156 (2005) $492-495$.

[117] J. Grzymala-Busse, Learning from examples based on rough multisets, in: Proceedings of the 2nd International Symposium on Methodologies for Intelligent Systems, Charlotte, NC, USA, 1987, pp. 325-332.

[118] Y. Guo, A. Şengür, A novel image segmentation algorithm based on neutrosophic similarity clustering, Appl. Soft Comput. 25 (2014) $391-398$.

[119] Y. Guo, A. Şengür, NCM: neutrosophic c-means clustering algorithm, Pattern Recognit. 48 (2015) 2710-2724.

[120] Y. Guo, A. Şengür, NECM: neutrosophic evidential c-means clustering algorithm, Neural Comput. Appl. 26 (2015) $561-571$.

[121] Y. Guo, R. Xia, A. Șengür, K. Polat, A novel image segmentation approach based on neutrosophic c-means clustering and indeterminacy filtering, Neural Comput. Appl. (2017) in press.

[122] R.P. Gylys, Quantal sets and sheaves over quantales, Liet. Matem. Rink. 34 (1994) 9-31.

[123] Hamidzadeh, J., Zabihimayvan, M., Sadeghi, R. (2017) Detection of Web site visitors based on fuzzy rough sets, Soft Computing, in press.

[124] R.J. Hathaway, J.C. Bezdek, W. Pedrycz, A parametric model for fusing heterogeneous fuzzy data, IEEE Trans. Fuzzy Syst. 4 (1996) $1277-1282$.

[125] T. Herawan, M.M. Deris, J.H. Abawajy, A rough set approach for selecting clustering attribute, Knowl. Based Syst. 23 (2010) $220-231$.

[126] C.-W. Huang, K.-P. Lin, M.-Ch. Wu, K.-C. Hung, G.-Shi. Liu, C.-H. Jen, Intuitionistic fuzzy c -means clustering algorithm with neighborhood attraction in segmenting medical image, Soft Comput. Fusion Found. Methodol. Appl. 19 (2015) 459-470.

[127] H.-L. Huang, New distance measure of single-valued neutrosophic sets and its application, Int. J. Intell. Syst. 31 (2016) $1021-1032$.

[128] W.L. Hung, M.S. Yang, Fuzzy clustering on LR-type fuzzy numbers with an application in Taiwanese tea evaluation, Fuzzy Sets Syst. 150 (2005) $561-577$.

[129] W.-L. Hung, J.-S. Lee, C.-D. Fuh, Fuzzy clustering based on based on intuitionistic fuzzy relations, Int. J. Uncertainty Fuzziness Knowledge-Based Syst. 12 (2004) 513-529.

[130] C. Hwang, F. Rhee, An interval type-2 fuzzy perceptron, Proc. Int. Conf. Fuzzy Syst. 2 (2002) 1331-1335.

[131] C. Hwang, F. Rhee, An interval type-2 fuzzy C spherical shells algorithm, Proc. Int. Conf. Fuzzy Syst. 2 (2004) 1117-1122.

[132] K.U. Jahn, Intervall-wertige Mengen, Math. Nach. 68 (1975) 115-132.

[133] A. Jain, R. Dubes, Algorithms for Clustering Data, Prentice-Hall, Inc, 1988.

[134] Z. Ji, Y. Xia, Q. Sun, G. Cao, Interval-valued possibilistic fuzzy C-means clustering algorithm, Fuzzy Sets Syst. 253 (2014) 138-156.

[135] J. Zhou, W. Pedrycz, D. Miao, Shadowed sets in the characterization of rough-fuzzy clustering, Pattern Recognit. 44 (2011) 1738-1749.

[136] M. Joshi, P. Lingras, Evidential clustering or rough clustering: the choice is yours, in: Rough Sets and Knowledge Technology, vol. 7414 of LNCS, 2012, 2012, pp. 123-128.

[137] J. Kacprzyk, J.W. Owsiński, D.A. Viattchenin, S. Shyrai, A new heuristic algorithm of possibilistic clustering based on intuitionistic fuzzy relations advances in intelligent systems and computing, in: 14th International Workshop on Intuitionistic Fuzzy Sets and Generalized Nets, IWIFSGN 2015, 26-28 October 2015, 401, Cracow, Poland, Springer, 2016, pp. 199-214.

[138] M.R.N. Kalhori, M.H.F. Zarandi, Interval type-2 credibilistic clustering for pattern recognition, Pattern Recognit. 48 (2015) (2015) $3652-3672$.

[139] O. Kanjanatarakul, S. Sriboonchitta, T. Denoeux, K-EVCLUS: Clustering large dissimilarity data in the belief function framework, in: Lecture Notes in Computer Science, 4th International Conference on Belief Functions: Theory and Applications, BELIEF 2016, 21-23 September 2016, , Prague, Czech Republic, Springer, 2016, pp. 105-112.

[140] S.R. Kannan, R. Devi, S. Ramathilagam, T.P. Hong, Effective fuzzy possibilistic c-means: an analyzing cancer medical database, Soft Comput. (2017) in press.

[141] F. Karaaslan, Correlation coefficients of single-valued neutrosophic refined soft sets and their applications in clustering analysis, Neural Comput. Appl. (2017) in press.

[142] N. Karthikeyani Visalakshi, K. Thangavel, R. Parvathi, An intuitionistic fuzzy approach to distributed fuzzy clustering, Int. J. Comput. Theory Eng. 2 (2010) 295-302.

[143] A. Kaushik, A.K. Soni, R. Soni, An improved functional link artificial neural networks with intuitionistic fuzzy clustering for software cost estimation, Int. J. Syst. Assur. Eng. Manag. 7 (2016) 50-61.

[144] E.E. Kerre, B. Reusch, K-H. Temme, A first view on the alternatives of fuzzy set theory, in: Computational Intelligence in Theory and Practice, Physica-Verlag, Heidelberg, 2001, pp. 55-72.

[145] K. Kishida, High-speed rough clustering for very large document collections, J. Am. Soc. Inf. Sci. Technol. 61 (2010) $1092-1104$.

[146] E. Klement, M. Puri, D. Ralescu, Limit theorems for fuzzy random variables, Proc. Roy. Soc. London Ser. A 407 (1986) $171-182$.

[147] G. Klir, Uncertainty and Information - Foundations of Generalized Information Theory, John Wiley and Sons, New Jersey, 2006.

[148] G.J. Klir, On fuzzy-set interpretation of possibility theory, Fuzzy Sets Syst. 108 (1999) $263-273$.

[149] D. Koundal, S. Gupta, S. Singh, Automated delineation of thyroid nodules in ultrasound images using spatial neutrosophic clustering and level set, Appl. Soft Comput. 40 (2016) 86-97.

[150] R. Krishnapuram, A. Joshi, O. Nasraoui, L. Yi, Low-complexity fuzzy relational clustering algorithms for web mining, IEEE Trans. Fuzzy Syst. 9 (4) (2001) 595-607.

[151] R. Krishnapuram, J. Keller, A possibilistic approach to clustering, IEEE Trans. Fuzzy Syst. 1 (1993) 98-110.

[152] R. Krishnapuram, J. Keller, The possibilistic c-means algorithm: insights and recommendations, IEEE Trans. Fuzzy Syst. 4 (1996) $385-393$.

[153] R. Kruse, K.D. Meyer, Statistics with Vague Data, Kluwer, Dordrecht, 1987.

[154] H. Kwakernaak, Fuzzy random variables-I, Inf. Sci. 15 (1978) 1-29.

[155] S. Kumar De, P.R. Krishna, Clustering web transactions using rough approximation, Fuzzy Sets Syst. 48 (2004) 131-138. 
[156] P. Kumar, P.R. Krishna, R.S. Bapi, S. Kumar De, Rough clustering of sequential data, Data Knowl. Eng. 63 (2007) 183-199.

[157] H. Kwakernaak, Fuzzy random variables-II, Inf. Sci. 17 (1979) 253-278.

[158] J.Z.C. Lai, E.Y.T. Juan, F.J.C. Lai, Rough clustering using generalized fuzzy clustering algorithm, Pattern Recognit. 46 (2013) $2538-2547$.

[159] J. Lake, Sets, fuzzy sets, multisets and functions, J. London Math. Soc., II Ser. 12 (1976) 323-326.

[160] J.M. Łęski, Robust possibilistic clustering, Arch. Control Sci. 10 (2000) 141-155.

[161] F. Li, M. Ye, X. Chen, An extension to Rough c-means clustering based on decision-theoretic Rough Sets model, Int. J. Approximate Reasoning 55 (2014) 116-129.

[162] M. Li, S. Deng, L. Wang, S. Feng, J. Fan, Hierarchical clustering algorithm for categorical data using a probabilistic rough set model, Knowl. Based Syst. 65 (2014) 60-71.

[163] X. Li, B. Liu, A sufficient and necessary condition for credibility measures, Int. J. Uncertainty Fuzziness Knowledge based Syst. 14 (2006) 527-535.

[164] X. Li, F. Wang, A clustering-based evidence reasoning method, Int. J. Intell. Syst. 31 (2016) 698-721.

[165] K.-P. Lin, A novel evolutionary kernel intuitionistic fuzzy c -means clustering algorithm institute of electrical and electronics engineers, IEEE Trans. Fuzzy Syst. 22 (2014) 1074-1087.

[166] O. Linda, M. Manic, General type-2 fuzzy c-means algorithm for uncertain fuzzy clustering, IEEE Trans. Fuzzy Syst. 20 (2012) 883-897.

[167] P. Lingras, Applications of rough set based k-means, Kohonen SOM, GA clustering, in: Transactions on Rough Sets VII, 2007, pp. 120-139.

[168] P. Lingras, F. Haider, M. Triff, Granular meta-clustering based on hierarchical, network, and temporal connections, Granular Comput. 1 (2016) 71-92.

[169] P. Lingras, G. Peters, Rough clustering, Wiley Interdiscip. Rev. 1 (2011) 64-72.

[170] P. Lingras, C. West, Interval Set Clustering of Web Users With Rough K-Means, Department of Mathematics and Computer Science, St. Mary's University, Halifax, Canada, 2002 Tech. Rep. 2002-002.

[171] P. Lingras, C. West, Interval set clustering of web users with rough k-means, J. Intell. Inf. Syst. 23 (2004) 5-16.

[172] P. Lingras, R. Yan, C. West, Comparison of conventional and rough k-means clustering, in: Proceedings RSFDGrC - 9th International Conference on Rough Sets, Fuzzy Sets, Data Mining and Granular Computing, LNAI, vol. 2639, Springer-Verlag, Berlin, Germany, 2003, pp. 130-137.

[173] B. Liu, Theory and Practice of Uncertain Programming, Physica-Verlag, Heidelberg, 2002.

[174] B. Liu, Uncertainty Theory: An Introduction to Its Axiomatic Foundations, Springer Verlag, Berlin, 2004.

[175] B. Liu, A survey of credibility theory, Fuzzy Optim. Decis. Making 5 (2006) 43-63.

[176] B. Liu, Y.-K. Liu, Expected value of fuzzy variable and fuzzy expected value models, IEEE Trans. Fuzzy Syst. 10 (4) (2002) 445-450.

[177] L.J. Liu, Y. Fu, S.W. Ma, R. Hu, Spatial load forecasting of distribution network based on intuitionistic fuzzy entropy and fuzzy clustering, Adv. Mater. Res. 516-517 (2012) 1433-1436.

[178] Z.-G. Liu, J. Dezert, G. Mercier, Q. Pan, Belief C-Means: an extension of Fuzzy C-Means algorithm in belief functions framework, Pattern Recognit. Lett. 33 (2012) 291-300.

[179] Z.-G. Liu, Q. Pan, J. Dezert, G. Mercier, Credal c-means clustering method based on belief functions, Knowledge-Based Syst. 74 (2015) 119-132.

[180] W. Ma, L. Jiao, M. Gong, C. Li, Image change detection based on an improved rough fuzzy c-means clustering algorithm, Int. J. Mach. Learn. Cybern. 5 (2014) 369-377.

[181] L. Maciel, R. Ballini, F. Gomide, Evolving possibilistic fuzzy modelling, J. Stat. Comput. Simul. 87 (2017) 1446-1466.

[182] P. Maji, Fuzzy-rough supervised attribute clustering algorithm and classification of microarray data, IEEE Trans. Syst. Man Cybern. Part B (Cybernetics) 41 (2011) 222-233.

[183] P. Maji, S.K. Pal, RFCM: a hybrid clustering algorithm using rough and fuzzy sets, Fundamenta Informaticae 80 (2007) $475-496$.

[184] P. Maji, S.K. Pal, Rough set based generalized fuzzy c-means algorithm and quantitative indices, IEEE Trans. Syst. Man Cybern. - Part B 37 (2007) $1529-1540$.

[185] P. Maji, S.K. Pal, Maximum class separability for rough-fuzzy c-means based brain MR image segmentation, Trans. Rough Sets IX (2008) 114-134.

[186] P. Maji, S. Paul, Microarray time-series data clustering using rough-fuzzy c-means algorithm, in: IEEE International Conference on Bioinformatics and Biomedicine, Atlanta, Georgia, 2011, pp. 269-272.

[187] P. Maji, S. Paul, Rough-fuzzy c-means for clustering microarray gene expression data, Perception and Machine Intelligence, LNCS. 7143, Springer, 2012.

[188] P. Maji, S. Paul, Rough-fuzzy clustering for grouping functionally similar genes from microarray data, IEEE/ACM Trans. Comput. Biol. Bioinf. 10 (2013) 286-299.

[189] D. Malyszko, J. Stepaniuk, Rough entropy based k-means clustering, in: Rough Sets, Fuzzy Sets, Data Mining and Granular Computing, vol. 5908 of LNCS, 2009, pp. 406-413.

[190] M.-H. Masson, T. Denœux, ECM: an evidential version of the fuzzy c-means algorithm, Pattern Recognit. 41 (2008) 1384-1397.

[191] M.-H. Masson, T. Denœux, RECM: relational evidential c-means algorithm, Pattern Recognit. Lett. 30 (2009) 1015-1026.

[192] M.-H. Masson, T. Denœux, Ensemble clustering in the belief functions framework, Int. J. Approximate Reasoning 52 (2011) $92-109$.

[193] G.J. McLachlan, S.I. Rathnayake, Mixture models for standard p-dimensional Euclidean data, in: C. Hennig, M. Meila, F. Murtagh, R. Rocci (Eds.), Handbook of Cluster Analysis, Chapman \& Hall, 2015, pp. 145-172.

[194] M. Ménard, V. Courboulay, P.A. Dardignac, Possibilistic and probabilistic fuzzy clustering: unification within the framework of the non-extensive thermostatistics, Pattern Recognit. 36 (2003) 1325-1342.

[195] J.M. Mendel, Uncertain Rule-Based Fuzzy Logic Systems: Introduction and New Directions, Prentice-Hall, Upper Saddle River, N.J., 2001.

[196] D. Meng, X. Zhang, K. Qin, Soft rough fuzzy sets and soft fuzzy rough sets, Comput. Math. Appl. 62 (2011) $4635-4645$.

[197] H.B. Michell, Pattern recognition using type-II fuzzy sets, Inf. Sci. 170 (2005) 409-418.

[198] J.H. Min, E.A. Shim, F.C.H. Rhee, An interval type-2 fuzzy PCM algorithm for pattern recognition, in: Proceedings of the 18th International Conference on Fuzzy Systems, FUZZ-IEEE'09, 2009, pp. 480-483.

[199] S. Mitra, An evolutionary rough partitive clustering, Pattern Recognit. Lett. 25 (2004) 1439-1449.

[200] S. Mitra, Computational intelligence in bioinformatics, Trans. Rough Sets III (2005) 134-152.

[201] Mitra, S., Barmann, B. (2008) Rough-fuzzy clustering: an application to medical imagery, in: RSKT 2008. No. 5009 in LNAI, 300-307.

[202] S. Mitra, P.P. Kundu, Satellite image segmentation with Shadowed C-means, Inf. Sci. 181 (2011) 3601-3613.

[203] S. Mitra, S.K. Pal, M.A. Siddiqi, Non-convex clustering using expectation maximization algorithm with rough set initialization, Pattern Recognit. Lett. (2003) 863-873.

[204] S. Mitra, W. Pedrycz, B. Barman, Shadowed c-means: integrating fuzzy and rough clustering, Pattern Recognit. 43 (2010) $1282-1291$.

[205] S. Mitra, W. Pedrycz, D. Miao, Shadowed sets in the characterization of rough-fuzzy clustering, Pattern Recognit. 44 (2011) 1738-1749.

[206] S. Mitra, H. Banka, W. Pedrycz, Rough-fuzzy collaborative clustering, IEEE Trans. Syst. Man Cybern. Part B (Cybernetics) 36 (2006) 795-805.

[207] S. Miyamoto, H. Ichihashi, K. Honda, Algorithms For Fuzzy Clustering - Methods in C-Means Clustering with Applications, Springer-Verlag, Berlin, 2008.

[208] M. Mizumoto, K. Tanaka, Some properties of fuzzy sets of type-2, Inf. Control 31 (1976) 312-340.

[209] M. Mizumoto, K. Tanaka, Fuzzy sets of type-2 under algebraic product and algebraic sum, Fuzzy Sets Syst 5 (1981) (1981) 277-290.

[210] O. Molodtsov, Soft set theory - first results, Comput. Math. Appl. 37 (1999) 19-31.

[211] R.E. Moore, Interval Analysis, Prentice-Hall, New York, 1966.

[212] A. Nakamura, Fuzzy rough sets, Notes Multiple-valued Logic Japan 9 (1988) 1-8.

[213] Narinyani, A.S. (1980) Underdetermined Sets - A new datatype for knowledge representation, Preprint 232, Project VOSTOK, 4, Novosibirsk, Computing Center, USSR Academy of Sciences.

[214] R.K. Nayak, D. Mishra, K. Shaw, S. Mishra, Rough set based attribute clustering for sample classification of gene expression data, Procedia Eng. 38 (2012) 1788-1792 
[215] T. Nguyen, S. Nahavandi, Modified AHP for gene selection and cancer classification using type-2 fuzzy logic, IEEE Trans. Fuzzy Syst. 24 (2016) $273-287$.

[216] M.R. Niakan, M.H.F. Zarandi, I.B. Turksen, A new credibilistic clustering algorithm, Inf. Sci. 279 (2014) 105-122.

[217] F. Pacheco, M. Cerrada, R.-V. Sánchez, D. Cabrera, C. Li, J.V. de Oliveira, Attribute clustering using rough set theory for feature selection in fault severity classification of rotating machinery, Expert Syst. Appl. 71 (2017) 69-86.

[218] N.R. Pal, K. Pal, J.M. Keller, J.C. Bezdek, A possibilistic fuzzy c-means clustering algorithm, IEEE T. Fuzzy Syst. 13 (2005) 517-530.

[219] I.-K. Park, G.-S. Choi, Rough set approach for clustering categorical data using information-theoretic dependency measure, Inf. Syst. 48 (2015) 289-295.

[220] D. Parmar, T. Wu, J. Blackhurst, MMR: an algorithm for clustering categorical data using Rough Set Theory, Data Knowl. Eng. 63 (2007) 879-893.

[221] Z. Pawlak, Rough sets, Int. J. Parallel Program. 11 (1982) 341-356.

[222] Z. Pawlak, Rough Sets: Theoretical Aspects of Reasoning About Data, Kluwer Academic Publishing, Dordrecht, 1991.

[223] Z. Pawlak, J. Grzymala-Busse, R. Slowinski, W. Ziarko, Rough Sets, Commun. ACM 38 (1995) 88-95.

[224] W. Pedrycz, Granular Computing. Analysis and Design of Intelligent Systems, CRC Press, Taylor and Francis Group, 2013.

[225] W. Pedrycz, Shadowed sets: representing and processing fuzzy sets, IEEE Trans. Syst. Man Cybern. - Part B 28 (1998) 103-109.

[226] W. Pedrycz, Interpretation of clusters in the framework of shadowed sets, Pattern Recognit. Lett. 26 (2005) 2439-2449.

[227] W. Pedrycz, Knowledge-Based Clustering: From Data to Information Granules, Wiley, Hoboken, NJ, 2005.

[228] W. Pedrycz, Granular computing-the emerging paradigm, J Uncertain Syst. 1 (2007) 38-61.

[229] W. Pedrycz, From fuzzy sets to shadowed sets: interpretation and computing, Int. J. Intell. Syst. 24 (2009) 48-61.

[230] W. Pedrycz, Shadowed sets in the characterization of rough-fuzzy clustering, Pattern Recognit. 44 (2011) 1738-1749.

[231] W. Pedrycz, R. Al-Hmouz, A.S. Balamash, A. Morfeq, Hierarchical granular clustering: an emergence of information granules of higher type and higher order, IEEE Trans. Fuzzy Syst. 23 (2015) 2270-2283

[232] W. Pedrycz, A. Bargiela, Granular clustering: a granular signature of data, IEEE Trans. Syst. Man Cybern. -Part B 32 (2002) $212-224$.

[233] W. Pedrycz, A. Skowron, V. Kreinovich (Eds.), Handbook of Granular Computing, Wiley, Chichester, 2008.

[234] N. Pelekis, D.K. Iakovidis, E.E. Kotsifakos, I. Kopanakis, Fuzzy clustering of intuitionistic fuzzy data, Int. J. Bus. Intell. Data Mining 3 (2008) 45-65.

[235] G. Peters, Outliers in rough k-means clustering, in: Proceedings PreMI 2005 - 1st International Conference on Pattern Recognition and Machine Intelligence. LNCS, vol. 3776, Springer-Verlag, Berlin, Germany, 2005, pp. 702-707.

[236] G. Peters, Some refinements of rough -means clustering, Pattern Recognit. 39 (2006) 1481-1491.

[237] G. Peters, Rough clustering utilizing the principle of indifference, Inf. Sci. 277 (2014) 358-374.

[238] G. Peters, F. Crespo, P. Lingras, R. Weber, Soft clustering - Fuzzy and rough approaches and their extensions and derivatives, Int. J. Approximate Reasoning 54 (2013) 307-322.

[239] G. Peters, M. Lampart, A partitive rough clustering algorithm, in: Proceedings RSCTC 2006 - 5th International Conference on Rough Sets and Current Trends in Computing. LNAI, vol. 4259, Springer-Verlag, Berlin, Germany, 2006, pp. 657-666.

[240] G. Peters, M. Lampart, R. Weber, Evolutionary rough k-medoids clustering, Trans. Rough Sets VIII (2008) (2008) 289-306.

[241] G. Peters, R. Weber, DCC: a framework for dynamic granular clustering, Granular Comput. 1 (2016) 1-11.

[242] G. Peters, R. Weber, R. Nowatzke, Dynamic rough clustering and its applications, Appl. Soft Comput. 12 (2012) 3193-3207.

[243] V.N. Pham, L.T. Ngo, W. Pedrycz, Interval-valued fuzzy set approach to fuzzy co-clustering for data classification, Knowledge-Based Syst. 107 (2016) $1-13$.

[244] C. Prabu, S.V.M.G. Bavithiraja, S. Narayanamoorthy, A novel brain image segmentation using intuitionistic fuzzy c means algorithm, Int. J. Imaging Syst. Technol. 26 (2016) 24-28.

[245] M.L. Puri, D.A. Ralescu, Fuzzy random variables, J. Math. Anal. Appl. 114 (1986) 409-422.

[246] M. Quafafou, $\alpha$-RST: a generalization of rough set theory, Inf. Sci. 124 (1-4) (2000) 301-316.

[247] F. Questier, I. Arnaut-Rollier, B. Walczak, D.L. Massart, Application of rough set theory to feature selection for unsupervised clustering, Chemom. Intell. Lab. Syst. 63 (2002) 155-167.

[248] T. Radecki, Level fuzzy sets, J. Cybern. (1977) 7.

[249] A.M. Radzikowska, E.E. Kerre, On L-Fuzzy Rough Sets, Artificial Intelligence and Soft Computing - ICAISC 2004, in: 7th International Conference, Zakopane, Poland, June 7-11, 2004, Proceedings; 01/2004, 2004.

[250] P. Rangasamy, S. Hadjitodorov, K. Atanassov, P. Vassilev, Generalized net model of an intuitionistic fuzzy clustering technique for biomedical data, Biotechnol. Biotechnol. Equip. 26 (2012) 3306-3309.

[251] F. Rhee, Uncertain fuzzy clustering: insights and recommendations, IEEE Comput. Intell. Mag. 2 (2007) 44-56.

[252] F. Rhee, C. Hwang, A type-2 fuzzy C-means clustering algorithm, in: Proc. Joint Conf. Int. Fuzzy Syst. Assoc./North Am. Fuzzy Inf. Process. Soc., 2001, pp. 1926-1929.

[253] F. Rhee, C. Hwang, An interval type-2 fuzzy K-nearest neighbor, Proc. Int. Conf. Fuzzy Syst. 2 (2003) 802-807.

[254] F. Rhee, C. Hwang, Uncertain fuzzy clustering: interval type-2 fuzzy approach to C-means, IEEE Trans. Fuzzy Syst. 15 (2007) $107-120$.

[255] R.M. Rodrìguez, L. Martìnez, V. Torra, Z.S. Xu, F. Herrera, Hesitant fuzzy sets: state of the art and future directions, Int. J. Intell. Syst. 29 (2014) 495-524.

[256] E. Rubio, O. Castillo, Designing type-2 fuzzy systems using the interval type-2 fuzzy c-means algorithm, in: Studies in Computational Intelligence, Recent Advances on Hybrid Approaches for Designing Intelligent Systems 2014, Springer, 2015, pp. 37-50.

[257] E. Rubio, O. Castillo, Interval type-2 fuzzy possibilistic c-means optimization using particle swarm optimization, in: Studies in Computational Intelligence, Nature-Inspired Design of Hybrid Intelligent Systems 2017, Springer, 2017, pp. 63-78.

[258] E. Rubio, O. Castillo, P. Melin, Interval type-2 fuzzy possibilistic c-means clustering algorithm, recent developments and new direction in soft-computing foundations and applications, Stud. Fuzziness Soft Comput. (2016) 185-194.

[259] E. Rubio, O. Castillo, P. Melin, Interval type-2 fuzzy system design based on the interval type-2 fuzzy c-means algorithm, in: Studies in Fuzziness and Soft Computing, Fuzzy Technology 2016, Springer, 2016, pp. 133-146.

[260] E. Ruspini, A new approach to clustering, Inf. Control 15 (1969) 22-32.

[261] E. Ruspini, Numerical methods for fuzzy clustering, Inf. Sci. 2 (1970) 319-350.

[262] E. Ruspini, New experimental results in fuzzy clustering, Inf. Sci. 6 (1973) 273-284.

[263] R. Sambuc, Fonctions $\varphi$-floues: Application a l'aide au diagnostic en pathologie thyroidienne Ph. D. Thesis, Univ. Marseille, France, 1975.

[264] M.A. Sanchez, O. Castillo, J.R. Castro, Information granule formation via the concept of uncertainty-based information with Interval Type-2 Fuzzy Sets representation and Takagi-Sugeno-Kang consequents optimized with Cuckoo search, Appl. Soft Comput. 27 (2015) 602-609.

[265] M.A. Sanchez, O. Castillo, J.R. Castro, P. Melin, Fuzzy granular gravitational clustering algorithm for multivariate data, Inf. Sci. 279 (2014) $498-511$.

[266] M. Sato, Y. Sato, Fuzzy clustering model for fuzzy data, in: Proceedings IEEE, 1995, pp. 2123-2128.

[267] L. Serir, E. Ramasso, N. Zerhouni, Evidential evolving Gustafson-Kessel algorithm for online data streams partitioning using belief function theory, Int. J. Approximate Reasoning 53 (2012) 747-768.

[268] G. Shafer, A Mathematical Theory of Evidence, Princeton University Press, 1976.

[269] J. Shan, H.D. Cheng, Y. Wang, A novel segmentation method for breast ultrasound images based on neutrosophic l-means clustering, Med. Phys. 3 (2012) 5669-5682.

[270] R. Shang, P. Tian, A. Wen, W. Liu, L. Jiao, An intuitionistic fuzzy possibilistic C-means clustering based on genetic algorithm, in: IEEE Congress on Evolutionary Computation, CEC 2016, 24-29 July, IEEE press, 2016, pp. 941-947.

[271] J. Shi, Y. Lei, Y. Zhou, M. Gong, Enhanced rough-fuzzy c-means algorithm with strict rough sets properties, Appl. Soft Comput. 46 (2016) 827-850.

[272] L. Shuai-Dong, C. Shi-Hong, Clustering of Web learners based on rough set, Wuhan Univ. J. Nat. Sci. 9 (2004) 542-546. 
[273] L. Silva, E. Moura, A.M.P. Canuto, R.H.N. Santiago, B. Bedregal, An interval-based framework for fuzzy clustering applications, IEEE Trans. Fuzzy Syst. 23 (2015) 2174-2186.

[274] F. Smarandache, Neutrosophy, Neutrosophic probability, Set, and Logic, American Research Press, Rehoboth, 1998.

[275] F. Smarandache, A Unifying Field in Logics Neutrosophic Logic. Neutrosophy, Neutrosophic Set, Neutrosophic Probability, American Research Press, Rehoboth, 2003.

[276] N.J.J. Smith, Vagueness and blurry sets, J. Phil. Logic 33 (2004) 165-235.

[277] L.H. Son, DPFCM: a novel distributed picture fuzzy clustering method on picture fuzzy sets, Expert Syst. Appl. 42 (2015) 51-66.

[278] L.H. Son, Generalized picture distance measure and applications to picture fuzzy clustering, Appl. Soft Comput. 46 (2016) $284-295$.

[279] L.H. Son, Measuring analogousness in picture fuzzy sets: from picture distance measures to picture association measures, Fuzzy Optim. Decis. Making (2017) in press.

[280] L.H. Son, B.C. Cuong, P.L. Lanzi, N.T. Thong, A novel intuitionistic fuzzy clustering method for geo-demographic analysis, Expert Syst. Appl. 39 (2012) 9848-9859.

[281] L.H. Son, P.H. Thong, Some novel hybrid forecast methods based on picture fuzzy clustering for weather nowcasting from satellite image sequences, Appl. Intell. 46 (2017) 1-15

[282] O. Takata, S. Miyamoto, K. Umayahara, Fuzzy clustering of data with uncertainties using minimum and maximum distances based on L1 metric, in: Proceedings of Joint 9th IFSA World Congress and 20th NAFIPS International Conference, July 25-28, 2001, Vancouver, British Columbia, Canada, 2001, pp. 2511-2516

[283] K.V. Thomas, L.S. Nair, Rough intuitionistic fuzzy sets in a lattice, Int. Math. Forum 6 (2011) $1327-1335$.

[284] N.T. Thong, L.H. Son, HIFCF: an effective hybrid model between picture fuzzy clustering and intuitionistic fuzzy recommender systems for medical diagnosis, Expert Syst. Appl. 42 (2015) 3682-3701.

[285] N.T. Thong, L.H. Son, Picture fuzzy clustering: a new computational intelligence method, Soft Comput. Fusion Found. Methodol. Appl. 20 (2016) 3549-3562.

[286] N.T. Thong, L.H. Son, A novel automatic picture fuzzy clustering method based on particle swarm optimization and picture composite cardinality, Knowledge-Based Syst. 109 (2016) 48-60.

[287] P. Thong, L.H. Son, Picture fuzzy clustering for complex data, Eng. Appl. Artif. Intell. 56 (2016) 121-130.

[288] L. Todorova, P. Vassilev, Algorithm for clustering data set represented by intuitionistic fuzzy estimates, Int. J. Bioautom. 14 (2010) 61-68.

[289] V. Torra, Hesitant fuzzy sets, Int. J. Intell. Syst. 25 (2010) 529-539.

[290] V.Y. Torra, Y. Narukawa, On hesitant fuzzy sets and decision, in: 18th IEEE International Conference on Fuzzy Sets, Jeju Island, Korea, 2009, pp. 1378-1382.

[291] V. Torra, L. Aliahmadipour, A. Dahlbom, Fuzzy, I-Fuzzy, and H-Fuzzy partitions to describe clusters, in: 2016 IEEE International Conference on Fuzzy Systems, FUZZ-IEEE 2016, 24-29 July 2016, Vancouver; Canada, IEEE press, 2016, pp. 524-530.

[292] V. Torra, S. Miyamoto, Y. Endo, J. Domingo-Ferrer, On intuitionistic fuzzy clustering for its application to privacy, in: Proceedings of the 2008 IEEE World Congress on Computational Intelligence (WCCI 2008) and the 17th IEEE International Conference on Fuzzy Systems (FUZZ-IEEE 2008), Hong Kong, China, IEEE Service Center, Piscataway, 2008, pp. 1042-1048.

[293] B.K. Tripathy, D. Mittal, D.P. Hudedagaddi, Hadoop with intuitionistic fuzzy C-means for clustering in big data, in: Advances in Intelligent Systems and Computing International Congress on Information and Communication Technology, ICICT 2015, 9-10 October 2015, Udaipur, India, Springer, 2016, pp. 599-610.

[294] B.K. Tripathy, A. Goyal, P.A. Sourav, A comparative analysis of rough intuitionistic fuzzy k-mode algorithm for clustering categorical data, Res. J. Pharm. Biol. Chem. Sci. 7 (2016) 2787-2802.

[295] V. Vapnik, The Nature of Statistical Learning, Springer-Verlag, New York, 1998.

[296] H. Verma, R.K. Agrawal, Possibilistic intuitionistic fuzzy c-means clustering algorithm for MRI brain image segmentation, Int. J. Artif. Intell. Tools 24 (2015) 24.

[297] H. Verma, R.K. Agrawal, A. Sharan, An improved intuitionistic fuzzy c-means clustering algorithm incorporating local information for brain image segmentation, Appl. Soft Comput. 46 (2016) 543-557.

[298] P. Viswanath, V.S. Babu, Rough-DBSCAN: a fast hybrid density based clustering method for large data sets, Pattern Recognit. Lett. 30 (2009) $1477-1488$.

[299] K.E. Voges, N.K. Pope, M.R. Brown, A rough cluster analysis of shopping orientation data, in: Proceedings of the Australian and New Zealand Marketing Academy Conference, Adelaide, 2003, 2003, pp. 1625-1631.

[300] K.E. Voges, N.K. Pope, M.R. Brown, Heuristics and optimization for knowledge discovery, in: Cluster Analysis of Marketing Data Examining On-Line Shopping Orientation: A Comparison of k-Means and Rough Clustering Approaches, Idea Group Publishing, Hershey, PA, 2002 , pp. 207-224.

[301] H. Wang, M. Zhou, A refined rough k-means clustering with hybrid threshold, in: Rough Sets and Current Trends in Computing, vol. 7413 of LNCS, 2012, pp. 26-35.

[302] 1. Wang, J. Wang, Feature weighting fuzzy clustering integrating rough sets and shadowed sets, Int. J. Pattern Recognit. Artif. Intell. 26 (4) (2012) 25.

[303] T. Wang, W.-L. Hung, A generalized possibilistic approach to shell clustering of template-based shapes, J. Stat. Comput. Simul. 87 (2017) 423-436.

[304] Z. Wang, Z. Xu, S. Liu, J. Tang, A netting clustering analysis method under intuitionistic fuzzy environment, Appl. Soft Comput. 11 (2011) 5558-5564.

[305] Z. Wang, Z. Xu, S. Liu, Z. Yao, Direct clustering analysis based on intuitionistic fuzzy implication, Appl. Soft Comput. 23 (2014) 1-8.

[306] W.G. Wee, On Generalizations of Adaptive Algorithms and Application of the Fuzzy Sets Concept to Pattern Classification PhD Thesis, Purdue University, Lafayette, 1967.

[307] P. Wen, L. Zheng, J. Zhou, Spatial credibilistic clustering algorithm in noise image segmentation, in: Proc. IEEE, 2007, pp. 543-547.

[308] P. Wen, J. Zhou, L. Zheng, A modified hybrid method of spatial credibilistic clustering and particle swarm optimization, Soft Comput. - Fusion Found. Methodol. Appl. 15 (2011) 855-865.

[309] T. Wu, X.-W. Liu, An interval type-2 fuzzy clustering solution for large-scale multiple-criteria group decision-making problems, Knowledge-Based Syst. 114 (2016) 118-127.

[310] X. Wu, B. Wu, J. Sun, H. Fu, Unsupervised possibilistic fuzzy clustering, J. Inf. Comput. Sci. 7 (2010) 1075-1080.

[311] S.D. Xenaki, K.D. Koutroumbas, A.A. Rontogiannis, A novel adaptive possibilistic clustering algorithm, IEEE Trans. Fuzzy Syst. 24 (2016) 791-810.

[312] D. Xu, Z. Xu, S. Liu, H. Zhao, A spectral clustering algorithm based on intuitionistic fuzzy information, Knowledge-Based Syst. 53 (2013) 20-26.

[313] Z. Xu, Intuitionistic fuzzy hierarchical clustering algorithms, J. Syst. Eng. Electron. 20 (2009) 1-8.

[314] Z. Xu, Intuitionistic Fuzzy Aggregation and Clustering, Springer-Verlag, Heidelberg, 2012

[315] Z. Xu, Hesitant Fuzzy Sets Theory, Springer Verlag, Heidelberg, 2014.

[316] Z. Xu, J. Chen, J. Wu, Clustering algorithm for intuitionistic fuzzy sets, Inf. Sci. 178 (2008) 3775-3790

[317] Z. Xu, J. Wu, Intuitionistic fuzzy C-means clustering algorithms, J. Syst. Eng. Electron. (IEEE) 21 (2010) 580-590.

[318] Z.S. Xu, M.M. Xia, Distance and similarity measures for hesitant fuzzy sets, Inf. Sci. 11 (2011) 2128-2138.

[319] Z. Xue, Y. Shang, A. Feng, Semi-supervised outlier detection based on fuzzy rough C-means clustering, Math. Comput. Simul. 80 (2010) 1911-1921.

[320] M. Yang, C. Ko, On a class of fuzzy c-numbers clustering procedures for fuzzy data, Fuzzy Sets Syst. 84 (1996) 49-60.

[321] M. Yang, H. Liu, Fuzzy clustering procedures for conical fuzzy vector data, Fuzzy Sets Syst. 106 (1999) $189-200$.

[322] M.-S. Yang, K.-L. Wu, Unsupervised possibilistic clustering, Pattern Recognit. 39 (2006) 5-21.

[323] I.T.R. Yanto, P. Vitasari, T. Herawan, M.M. Deris, Applying variable precision rough set model for clustering student suffering study's anxiety, Expert Syst. Appl. 39 (2012) 452-459.

[324] D. Yao, X. Liu, X. Zhang, C. Wang, Type-2 fuzzy cross-entropy and entropy measures and their applications, J. Intell. Fuzzy Syst. 30 (2016) 2169-2180. 
[325] Y.Y. Yao, Combination of rough and fuzzy sets based on $\alpha$-level sets, in: T.Y. Lin, N. Cercone (Eds.), Rough Sets and Data Mining: Analysis for Imprecise Data, Kluwer Academic Publishers, Boston, 1997, pp. 301-321.

[326] Y.Y. Yao, A comparative study of fuzzy sets and rough sets, Inf. Sci. 109 (1998) 227-242.

[327] J. Ye, Clustering methods using distance-based similarity measures of single-valued neutrosophic sets, J. Intell. Syst. 23 (2014) $379-389$.

[328] J. Ye, Single-valued neutrosophic minimum spanning tree and its clustering method, J. Intell. Syst. 23 (2014) $311-324$.

[329] J. Ye, A netting method for clustering-simplified neutrosophic information, Soft Comput. Fusion Found. Methodol. Appl. (2017) in press.

[330] B. Yu, Z. Niu, L. Wang, Mean shift based clustering of neutrosophic domain for unsupervised constructions detection, Optik - Int. J. Light Electron Optics 124 (2013) 4697-4706.

[331] L. Zadeh, Information granulation and its centrality in human and machine intelligence, in: G. Grahne (Ed.), Proceedings of the 6. Scandinavian conference on artificial intelligence (SCAI'97). Frontiers in artificial intelligence and applications, 40, Amsterdam, IOS Press, 1997, pp. 26-27.

[332] L.A. Zadeh, Fuzzy sets, Inf. Control 8 (1965) 338-353.

[333] L.A. Zadeh, Fuzzy sets as a basis for a theory of possibility, Fuzzy Sets Syst. 1 (1978) 3-28.

[334] M.H. Zarandi, Z.S. Razaee, A fuzzy clustering model for fuzzy data with outliers, Int. J. Fuzzy Syst. Appl. (IJFSA) 1 (2) (2011) $29-42$.

[335] J. Zhang, T. Li, H. Chen, Composite rough sets for dynamic data mining, Inf. Sci. 257 (2014) 81-100.

[336] J.-S Zhang, Y.-W. Leung, Improved possibilistic C-means clustering algorithms, IEEE T. Fuzzy Syst. 12 (2004) $209-217$.

[337] T. Zhang, L. Chen, C.L.P. Chen, Clustering algorithm based on spatial shadowed fuzzy C-means and I-Ching operators, Int. J. Fuzzy Syst. 18 (2016) 609-617.

[338] T. Zhang, L. Chen, F. Ma, A modified rough c-means clustering algorithm based on hybrid imbalanced measure of distance and density, Int. J. Approximate Reasoning 55 (2014) 1805-1818.

[339] W.-R. Zhang, L. Zhang, YinYang bipolar logic and bipolar fuzzy logic, Inf. Sci. 165 (2004) 265-287.

[340] W.-R. Zhang, (Yin)(Yang) Bipolar Fuzzy Sets, in: Proc. of IEEE World Congress on Computational Intelligence - Fuzz-IEEE, Anchorage, AK, May 1998, 1998, pp. 835-840.

[341] W.-R. Zhang, YinYang Bipolar Relativity: A Unifying Theory of Nature, Agents and Causality with Applications in Quantum Computing, Cognitive Informatics and Life Sciences, IGI Global, Hershey and New York, 20112011.

[342] X. Zhang, Z. Xu, An MST cluster analysis method under hesitant fuzzy environment, Control Cybern. 41 (2012) 645-666.

[343] X. Zhang, Z. Xu, Novel distance and similarity measures on hesitant fuzzy sets with applications to clustering analysis, J. Intell. Fuzzy Syst. 28 (2015) $2279-2296$.

[344] X. Zhang, Z. Xu, Hesitant fuzzy agglomerative hierarchical clustering algorithms, Int. J. Syst. Sci. 46 (2015) $562-576$.

[345] H. Zhao, Z. Xu, S Liu, Z. Wang, Intuitionistic fuzzy MST clustering algorithms, Comput. Indus. Eng. 62 (2012) 1130-1140.

[346] H. Zhao, Z. Xu, Z. Wang, Intuitionistic fuzzy clustering algorithm based on boole matrix and association measure, Int. J. Inf. Technol. Decis. Making 12 (2013) 95-118.

[347] J. Zhou, C.C. Hung, X. Wang, S. Chen, Fuzzy clustering based on credibility measure, in: Proceedings of the Sixth International Conference on Information and Management Sciences, Lhasa, China, 2007, pp. 404-411.

[348] J. Zhou, Q. Wang, C.-C. Hung, Z. Yi, Credibilistic clustering: the model and algorithms, Int. J. Uncertainty Fuzziness Knowledge Based Syst. 23 (2015) 545.

[349] J. Zhou, L. Zheng, X. Chen, B. Anderson, Hybrid method of spatial credibilistic clustering and particle swarm optimization: discussion and application, in: Proceedings of the IEEE Sixth International Conference on Fuzzy Systems and Knowledge Discovery, 2009, pp. 423-427.

[350] K. Zhou, A. Martin, Q. Pan, Z.-G. Liu, Median evidential c-means algorithm and its application to community detection, Knowledge-Based Syst. 74 (2015) 69-88.

[351] K. Zhou, A. Martin, Q. Pan, Z.-G. Liu, ECMdd: Evidential c-medoids clustering with multiple prototypes, Pattern Recognit. 60 (2016) $239-257$.

[352] X. Zhou, R. Zhao, F. Yu, H. Tian, Intuitionistic fuzzy entropy clustering algorithm for infrared image segmentation, J. Intell. Fuzzy Syst. 30 (2016) $1831-1840$.

[353] L. Zadeh, The concept of a linguistic variable and its application to approximate reasoning-I, Inf. Sci. 8 (1975) 199-249.

[354] R.R. Yager, On the theory of bags, Int. J. Gen. Syst. 13 (1986) 23-37.

[355] J.C. Bezdek, Pattern Recognition with Fuzzy Objective Function Algorithms, Plenum Press, New York, 1981.

[356] J.C. Dunn, A fuzzy relative of the ISODATA process and its use in detecting compact well-separated clusters, J. Cybern. 3 (1974) 32-57.

[357] M.-S. Yang, A survey of fuzzy clustering, Mathematical Comput, Modelling 18 (11) (1993) 1-16.

[358] Z. Xie, S. Wang, F.L. Chung, An enhanced possibilistic C-Means clustering algorithm EPCM, Soft Computing 12 (2008) $593-611$.

[359] C. Hwang, F. Rhee, Uncertain fuzzy clustering: interval Type-2 fuzzy approach to C-means, IEEE T. Fuzzy Syst. 15 (2007) 107-120.

[360] Y. Guo, A. Şengür, NCM: Neutrosophic c-means clustering algorithm, Pattern Recognit. 48 (2015) $2710-2724$.

[361] M. Burgin, A. Chunihin, Named Sets in the Analysis of Uncertainty, in Methodological and Theoretical Problems of Mathematics and Information Sciences, Kiev (1997) 72-85. 